EUROPEAN ORGANIZATION FOR NUCLEAR RESEARCH

CERN-EP/2003-051

18 August 2003

\title{
The ATHENA Antihydrogen Apparatus
}

The ATHENA Collaboration

\begin{abstract}
The ATHENA apparatus that recently produced and detected the first cold antihydrogen atoms is described. Its main features, which are described herein, are: an external positron accumulator, making it possible to accumulate large numbers of positrons; a separate antiproton catching trap, optimizing the catching, cooling and handling of antiprotons; a unique high resolution antihydrogen annihilation detector, allowing an clear determination that antihydrogen has been produced; an open, modular design making variations in the experimental approach possible and a "nested" Penning trap situated in a cryogenic, 3T magnetic field environment used for the mixing of the antiprotons and positrons.
\end{abstract}

Submitted to Nucl. Instr. Meth. A 


\title{
The ATHENA antihydrogen apparatus
}

M. Amoretti ${ }^{\text {a }}$, C. Amsler ${ }^{\mathrm{b}}$, G. Bonomi ${ }^{\mathrm{d}}$, A. Bouchta ${ }^{\mathrm{d}}$, P. D. Bowe ${ }^{\mathrm{e}}$, C. Carraro ${ }^{\mathrm{a}}$, M. Charlton ${ }^{\mathrm{f}}$, M. J. T. Collier ${ }^{\mathrm{f}}$, M. Doser ${ }^{\mathrm{d}}$, V. Filippini ${ }^{\mathrm{c}}$, K. S. Fine ${ }^{\mathrm{d}}$, A. Fontana ${ }^{\mathrm{c}}$, M. C. Fujiwara ${ }^{\mathrm{g}}$, R. Funakoshi ${ }^{\mathrm{g}}$, P. Genova ${ }^{\mathrm{c}}$, A. Glauser ${ }^{\mathrm{b}}$, D. Grögler ${ }^{\mathrm{b}}$, J. Hangst ${ }^{\mathrm{e}}$, R. S. Hayano ${ }^{\mathrm{g}}$, H. Higaki $^{\mathrm{g}}$, M. H. Holzscheiter ${ }^{\mathrm{d}, 1}$, W. Joffrain ${ }^{\mathrm{a}}$, L. V. Jørgensen ${ }^{\mathrm{f}, *}$, V. Lagomarsino ${ }^{\mathrm{a}}$, R. Landua ${ }^{\mathrm{d}}$, C. Lenz Cesar ${ }^{\mathrm{h}}$, D. Lindelöf ${ }^{\mathrm{b}}$, E. Lodi-Rizzini ${ }^{\mathrm{i}}$, M. Macri ${ }^{\mathrm{a}}$, N. Madsen ${ }^{\mathrm{b}}$, D. Manuzio ${ }^{\mathrm{a}}$, G. Manuzio ${ }^{\text {a }}$, M. Marchesotti ${ }^{\mathrm{d}}$, P. Montagna ${ }^{\mathrm{c}}, \mathrm{H}$. Pruys ${ }^{\mathrm{b}}$, C. Regenfus ${ }^{\mathrm{b}}$, P. Riedler ${ }^{\mathrm{d}}$, J. Rochet ${ }^{\mathrm{d}}$, A. Rotondi ${ }^{\mathrm{c}}$, G. Rouleau ${ }^{\mathrm{d}, 2}$, G. Testera ${ }^{a}$, D. P. van der $\operatorname{Werf}^{f}$, A. Variola ${ }^{a}$, T. L. Watson ${ }^{f}$, T. Yamazaki ${ }^{\mathrm{g}}$, Y. Yamazaki ${ }^{\mathrm{g}}$

The ATHENA Collaboration

${ }^{a}$ Genoa University \& INFN, Genoa, Italy

${ }^{\mathrm{b}}$ Inst. of Physics, Zurich University, Zurich, Switzerland

${ }^{\mathrm{c}}$ Pavia University \& INFN, Pavia, Italy

${ }^{\mathrm{d}}$ CERN, Geneva, Switzerland

e Dept. of Physics \& Astronomy, University of Aarhus, Aarhus, Denmark

${ }^{\mathrm{f}}$ Department of Physics, University of Wales Swansea, Wales, United Kingdom

${ }^{\mathrm{g}}$ Dept. of Physics and Inst. of Physics, Tokyo University, Tokyo, Japan

${ }^{\mathrm{h}}$ Fed. Univ. Rio de Janeiro (UFRJ), Rio de Janeiro, Brazil

${ }^{\mathrm{i} B r e s c i a}$ University \& INFN, Brescia, Italy

\begin{abstract}
The ATHENA apparatus that recently produced and detected the first cold antihydrogen atoms is described. Its main features, which are described herein, are: an external positron accumulator, making it possible to accumulate large numbers of positrons; a separate antiproton catching trap, optimizing the catching, cooling and handling of antiprotons; a unique high resolution antihydrogen annihilation detector, allowing an clear determination that antihydrogen has been produced; an open, modular design making variations in
\end{abstract}


the experimental approach possible and a "nested" Penning trap situated in a cryogenic, 3T magnetic field environment used for the mixing of the antiprotons and positrons.

Key words: Antihydrogen, Penning trap, cryogenic detector, positrons, antiprotons

\section{Introduction}

The ATHENA collaboration recently succeeded in making the first cold antihydrogen $(\bar{H})$ by mixing antiprotons with a dense cold positron plasma [1]. This was an important milestone on the way to the main goal of the ATHENA experiment, which is a spectroscopic comparison of the properties of antihydrogen and hydrogen atoms. This will allow direct tests of CPT invariance for leptons and baryons with unprecedented accuracy. For example, the long lifetime of the metastable $2 \mathrm{~s}$ state $(122 \mathrm{msec})$ leads to a natural line width of about $1 \mathrm{~Hz}$ for the $1 \mathrm{~s}-2 \mathrm{~s}$ transition, offering, in principle, the possibility to compare anti-atoms with their matter counterparts with high precision, perhaps reaching 1 part in $10^{18}$. In fact, an accuracy close to $10^{-14}$ should be achievable for antihydrogen if the 2 photon $1 \mathrm{~s}$ to $2 \mathrm{~s}$ transition can be measured with a similar precision to that achieved for hydrogen in 2000 [2]. Such precision is several orders of magnitudes better than the present best direct CPT tests on leptons and baryons, and comparable to the indirect tests in the neutral kaon system [3].

The first observation of atomic antimatter was made in 1996 at CERN when the formation of antiatoms was reported at LEAR (Low Energy Antiproton Ring). The internal antiproton beam with a kinetic energy in the GeV range traversed a Xe gas jet target and produced $e^{+} e^{-}$pairs. In a very small fraction of these collisions a positron was captured by the antiprotons resulting in 9 events attributed to antihydrogen [4]. Similar observations were made in a subsequent experiment at Fermilab [5]. These antihydrogen atoms were all created at kinetic energies in the 1-6 GeV range corresponding to equilibrium temperatures in the $10^{13} \mathrm{~K}$ range. However, antihydrogen needs to be produced in much larger quantities and at much lower kinetic energies (i.e. so-called "cold" antihydrogen) to facilitate the aforementioned precision spectroscopic measurements of its properties. Indeed the highest precision is expected for measurements on the antihydrogen atom held at rest in a neutral atom magnetic trap. The recent results from ATHENA have brought spectroscopy

\footnotetext{
* Corresponding author.

Email address: Lars. Varming. Jorgensen@ cern. ch (L. V. Jørgensen). URL: http: / / athena . web. cern. ch/athena/ (L. V. Jørgensen).

1 Visitor from Los Alamos National Laboratory, USA

2 Now at Los Alamos National Laboratory, USA
} 
a large step closer by making copious amounts of cold antihydrogen available for experiments [1].

In the context of cold antihydrogen formation two mechanisms are of importance and their parameter dependence determines the boundary conditions for the design of any experiment to produce and manipulate cold antihydrogen. The spontaneous radiative recombination of positrons with antiprotons $\left(e^{+}+\bar{p} \rightarrow \bar{H}+h \nu\right)$ depends on the positron temperature $(\propto 1 / \sqrt{T})$ and the positron density $(\propto n)$ and results predominantly in atomic levels with low principal quantum numbers. Three-body recombination $\left(e^{+}+e^{+}+\bar{p} \rightarrow \bar{H}+e^{+}\right)$becomes competitive at cryogenic temperatures $\left(\propto 1 / T^{4.5}\right)$ and high positron densities $\left(\propto n^{2}\right)$ and mainly populates atomic levels with high principal quantum numbers. De-excitation to the ground state is slow and the nascent atom is susceptible to ionization by electric fields.

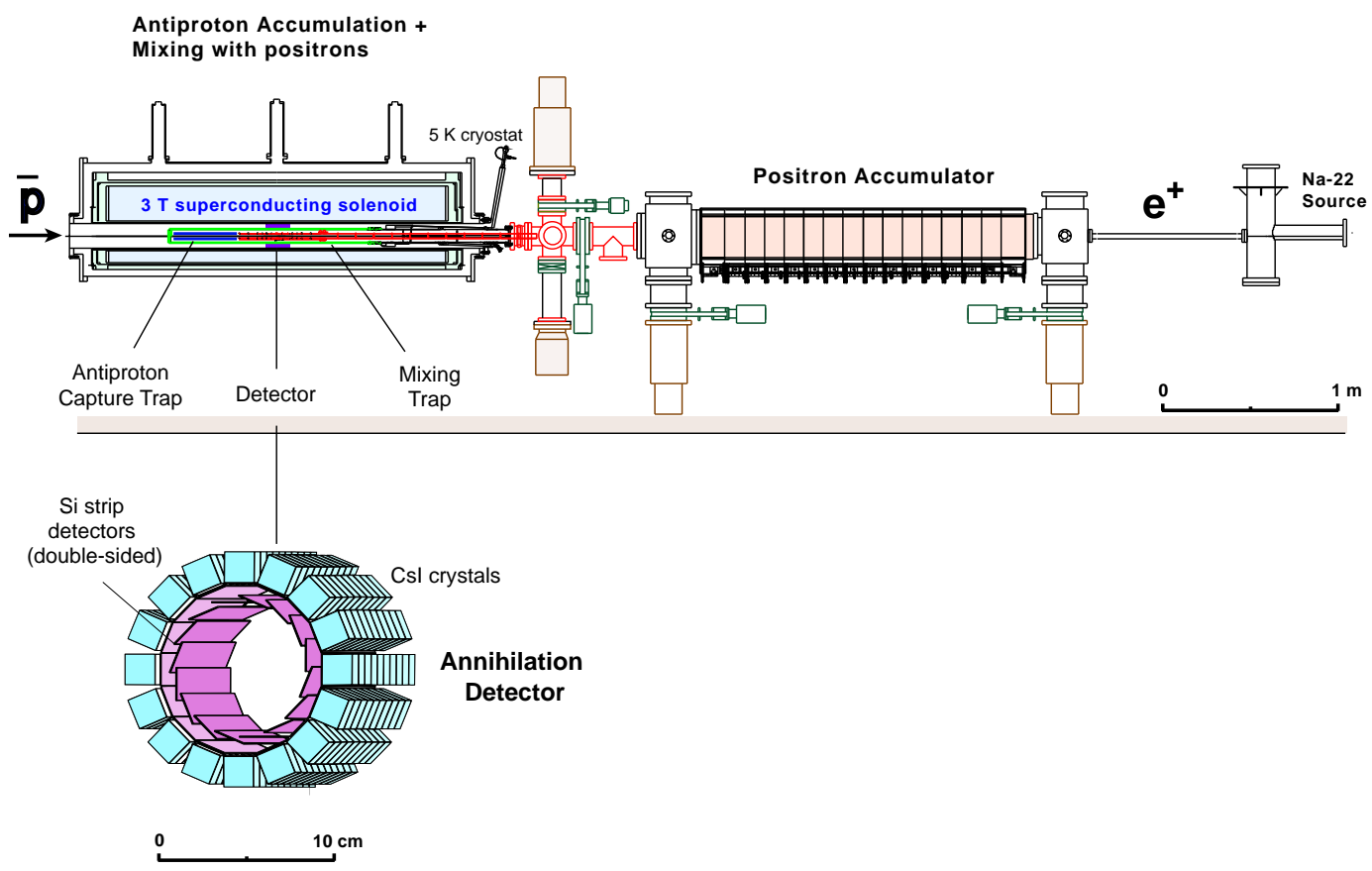

Fig. 1. Overview of the ATHENA apparatus for the production and detection of antihydrogen. An expanded view of the annihilation detector is shown below the main apparatus.

In the present paper we describe in detail the equipment used by ATHENA to produce and detect cold antihydrogen atoms. A schematic illustration of the apparatus is shown in Fig. 1. Low energy antiprotons are extracted from the CERN Antiproton Decelerator (AD). A superconducting solenoid (3 T) with a cold bore houses the antiproton capture trap and the antiproton-positron mixing trap. The antiprotons are moderated through a silicon beam-profiling counter, a foil and various windows and are then reflected in the catching trap by a high voltage electrode. About 500$700 \mathrm{~ns}$ after the arrival of the pulse a high voltage potential is raised on the entrance electrode to capture the antiproton bunch. They are then cooled inside the catching trap to meV energies by Coulomb interactions with a pre-loaded electron cloud. The antiprotons are confined in the radial direction by the magnetic field of the 
solenoid and in the axial direction by an electrostatic field produced by ten cylindrical electrodes having an inner diameter of $2.5 \mathrm{~cm}$. The field configuration is similar to the one used in Penning traps [6]. Further details can be found in sections 2,4 and 5.

The positrons from a $1.4 \mathrm{GBq}(40 \mathrm{mCi}){ }^{22} \mathrm{Na}$ source are moderated in solid neon and transferred into a longitudinal magnetic field region where they lose energy by collisions with nitrogen gas and are eventually confined in a system of cylindrical electrodes, as described in section 7. They are then transferred to the superconducting solenoid where they are held in a trap similar to the one used to store the antiprotons.

The particles stacked in the two traps are transported to the mixing trap held at a pressure below $10^{-12}$ mbar. The formation of antihydrogen atoms is studied by observing their annihilation when they impinge upon the electrodes of the mixing trap. The annihilation detector, a large solid angle array of silicon microstrip counters and CsI crystals, surrounds the mixing trap. It measures the charged hadron tracks (mostly pions) emitted by the annihilation of the antiproton, in temporal and spatial coincidence with the two back-to-back $511 \mathrm{keV}$ photons from $e^{+} e^{-}$annihilation. The detector is described in more detail in section 8 .

In section 9 we describe the control and data acquisition system and in section 10 the online software for time-ordering and rapid real time analysis of the data as it arrives is presented. Finally in section 11 the offline analysis software, Monte Carlo simulation of the apparatus and event reconstruction and selection schemes are described.

\section{General experimental parameters}

In the design of the ATHENA apparatus emphasis was placed on an open and flexible system. This has allowed for the accumulation of large numbers of positrons in an external apparatus with subsequent transfer into the main apparatus as well as limited freedom in the approach to antihydrogen formation and the types of experiments that could be performed. Due to the temperature dependence of both of the formation mechanisms mentioned in section 1 it is crucial to maintain the lowest possible temperature in the trapping region. In addition to this the two other main experimental conditions necessary for making antihydrogen are a very low residual gas pressure and a high magnetic field. 


\subsection{The superconducting magnet and the cryogenic cold nose}

The magnet used for the ATHENA experiment was taken over from the original PS200T system [7] and consists of a superconducting solenoid with a $150 \mathrm{~mm}$ diameter room temperature bore and a homogeneous field region of $1 \mathrm{~m}$ length. The magnet is capable of reaching $5 \mathrm{~T}$ but for the ATHENA experiments was generally operated at $3 \mathrm{~T}$. The requirements of the ATHENA experiment were significantly more complex than the antiproton trapping apparatus developed for PS200T such that a number of modifications to the original system were necessary.

To allow easy access to the trap without disturbing the cryogenic system of the magnet, a separate continuous flow cryostat (cold nose) was installed in the bore of the magnet to cool the vacuum shell housing the trap. The cold nose can be cooled to a few $\mathrm{K}$, improving the cryogenic pumping speed at the location of the traps. In addition, since the electrons and positrons cool very efficiently by synchrotron radiation in the $3 \mathrm{~T}$ field, a low ambient temperature allows production of very cold positron and electron plasmas and, via sympathetic cooling, also very cold antiprotons. The cold nose is closed off at the end toward the AD beamline by the degrader foils and the silicon beamcounter. At the end toward the positron accumulator it has an open connection to a room temperature vacuum chamber. During normal operation the outside of the cold nose needs to be shielded from room temperature radiation by lowering the bore temperature of the main magnet to. To allow radial space for mounting the trap, the trap vacuum system, the cold nose, and finally the detector, a common nitrogen shield for both the main magnet system and the cold nose was designed. Under normal operation a temperature of $130 \mathrm{~K}$ was achieved on the bore walls.

\subsection{The vacuum system}

Electromagnetic traps of the Penning and Penning-Malmberg types are used to store, cool and manipulate the charged particles required by ATHENA (positrons, antiprotons and the electrons necessary to cool the antiprotons). These traps (with the exception of the positron accumulator; see section 7) are all situated within the vacuum vessel of the cryogenic cold nose inside the superconducting solenoid. The trap environmental temperature is representative of the equilibrium temperature of the antiproton and electron clouds at the end of the cooling process. In addition, cooling the trap region reduces the surface outgassing and transforms all the surfaces of the apparatus into a cryogenic pump. The gas flow from the room temperature region is reduced by the pumping system installed in that region and by the trap and cold nose surfaces. Most of the walls in the room temperature section have been coated with a NEG (Non Evaporable Getter) material. After an initial pumpout this material is activated by raising the temperature of the walls to $200^{\circ} \mathrm{C}$ for 24 hours. 
This effectively turns the coated surfaces into vacuum pumps. In addition to this a special vacuum chamber filled with NEG coated metal strips was installed. The NEG coated surfaces together with a $300 \mathrm{ls}^{-1}$ ionpump constituted the ultra high vacuum pumping system in the room temperature region. The pressure can only be directly measured in the room temperature region, where $10^{-11}$ mbar is routinely achieved. The maximum temperature of the trap is approximately $15 \mathrm{~K}$ when the complete apparatus is installed inside the cold nose, though the walls of the cold nose itself reaches temperatures in the range $4-10 \mathrm{~K}$.

\section{Antiproton beam monitoring}

Pulses of antiprotons are ejected from the AD with a momentum of about 100 $\mathrm{MeV} / \mathrm{c}$ (or a kinetic energy of around 5.3 MeV)[8]. The $\bar{p}$ shots last for $200 \mathrm{~ns}$ and are repeated every $100 \mathrm{~s}$. Typically, $2 \times 10^{7}$ antiprotons are injected into the ATHENA apparatus. The locations of the beam detectors are sketched in Fig. 2. The antiproton kinetic energy is degraded by $25 \mu \mathrm{m}$ of stainless steel, located $10 \mathrm{~cm}$ upstream of the $\bar{p}$ catching trap, and by $130 \mu \mathrm{m}$ of aluminum on the entrance electrode.

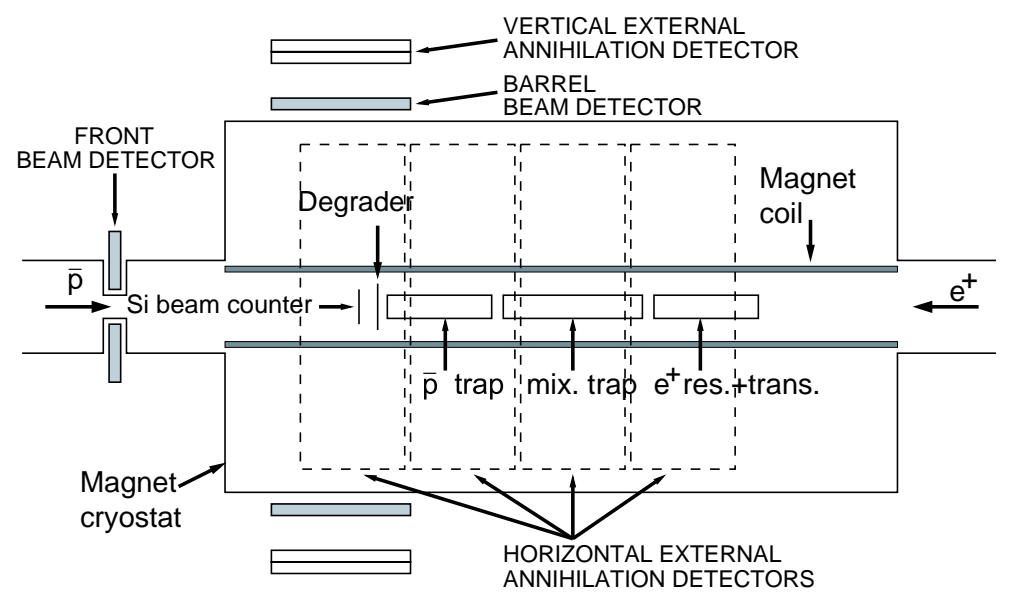

Fig. 2. Top view of the $\bar{p}$ beam line (not to scale). The dashed rectangles show the locations of the four External Annihilation Detectors below the apparatus.

The antiproton beam is monitored by three systems of detectors: (i) a silicon counter provides the $\bar{p}$ trigger for the catching trap high voltage switch and measures the beam profile; (ii) the external beam detector, which comprises the front and barrel plastic scintillators, detects the annihilation in a pulse mode and monitors the beam intensity and stability; (iii) the horizontal and vertical external annihilation detectors, consisting also of plastic scintillators, are used to study the trapped antiprotons by detecting in a single counting mode the pions following annihilation either on the residual gas or on the trap walls. 


\subsection{The silicon beam counter}

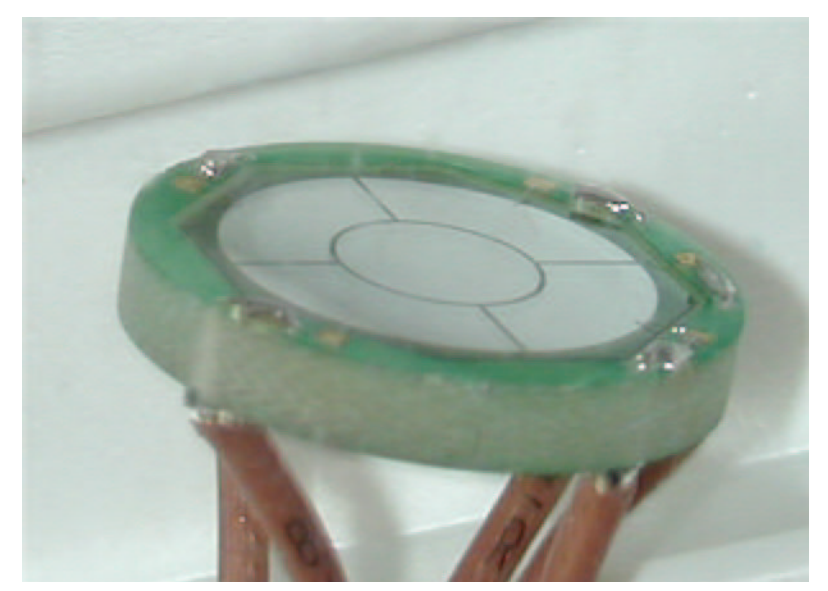

Fig. 3. Photograph of the $67 \mu \mathrm{m}$ thick silicon counter mounted in a PCB frame. The contacts to the individual pads are made using ultrasonic wire-bonds.

The silicon beam counter [9] is a $67 \mu \mathrm{m}$ thick silicon diode, $15 \mathrm{~mm}$ in diameter, that was originally designed for the Crystal Barrel experiment [10]. The diode (Fig. 3) is segmented in five pads, each connected to an individual signal line. The voltage required to fully deplete the diode is $4.5 \mathrm{~V}$. The beamcounter is located in front of the antiproton catching trap (see section 4.1) and is operated at temperatures ranging from $10 \mathrm{~K}$ to $300 \mathrm{~K}$, in a vacuum of $\approx 10^{-8}$ mbar and in a $3 \mathrm{~T}$ magnetic field. A very thin counter has to be used to allow maximum transmission of the low energy antiprotons into the catching trap. The average energy loss of $5.3 \mathrm{MeV}$ antiprotons in silicon was estimated from experimental data [11] to be about $11.4 \mathrm{keV}$ per micrometer of silicon, thus creating 3200 electron-hole (e-h) pairs, compared to 80 e-h pairs for minimum ionizing particles. Thus, around $3.2 \times 10^{10}$ charge pairs per micrometer of silicon are generated in the ATHENA silicon beamcounter for an antiproton beam intensity of $10^{7} \bar{p}$ per spill. In order to detect this high instanteneous current, a readout system was developed where the signal current is read directly across a $100 \Omega$ protection resistor and fed into a digital oscilloscope and an ADC. This also allows a direct measurement of the spill duration. The signal from the silicon beam counter is also used to trigger the $\bar{p}$-catching-trap. A typical signal registered on an oscilloscope is shown in Fig. 4.

Fig. 5 shows the fraction of the total signal measured on the central pad for different bias voltages. For each voltage setting the mean integrated signal of three antiproton spills is shown. The error bars indicate the standard deviation. The signal starts to plateau at about $30 \mathrm{~V}$ but an operating voltage of $100 \mathrm{~V}$ was chosen to achieve better timing performances and to reduce signal losses due to recombination of charge carriers. 


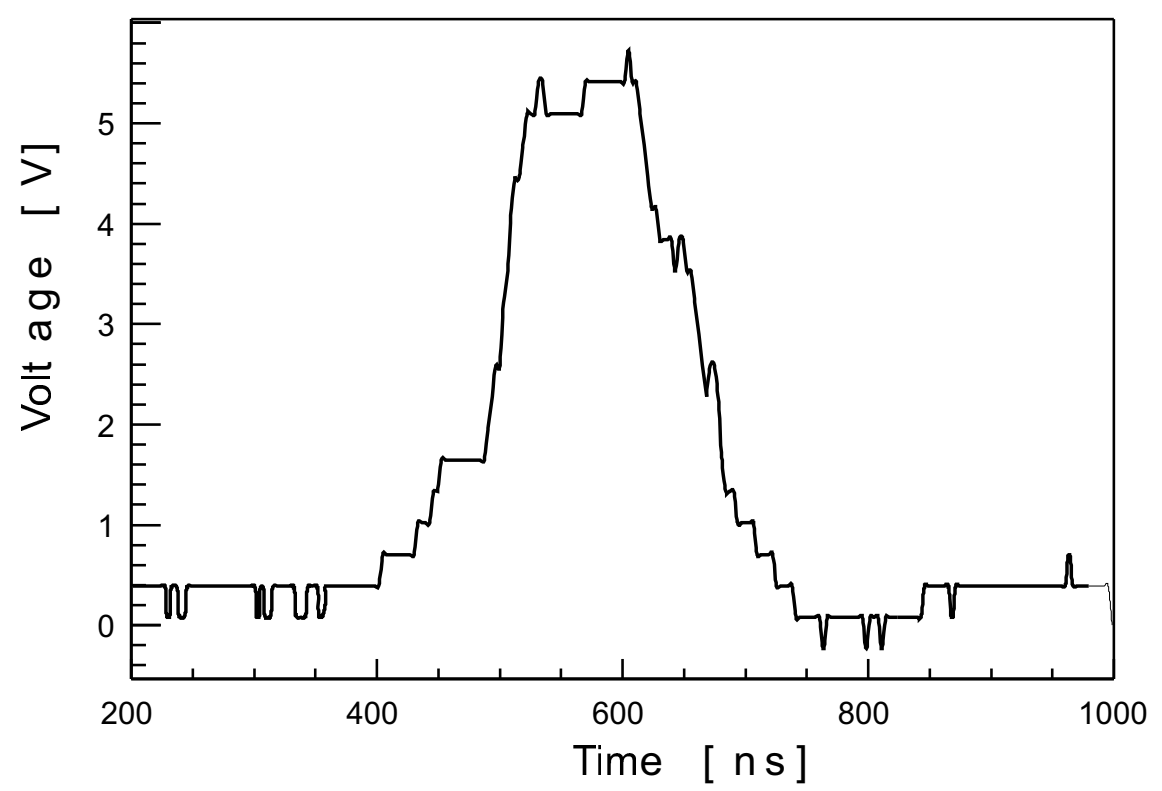

Fig. 4. Signal on the central pad of the silicon beam counter registered on an oscilloscope. The spill duration of around $200 \mathrm{~ns}$ is clearly visible.

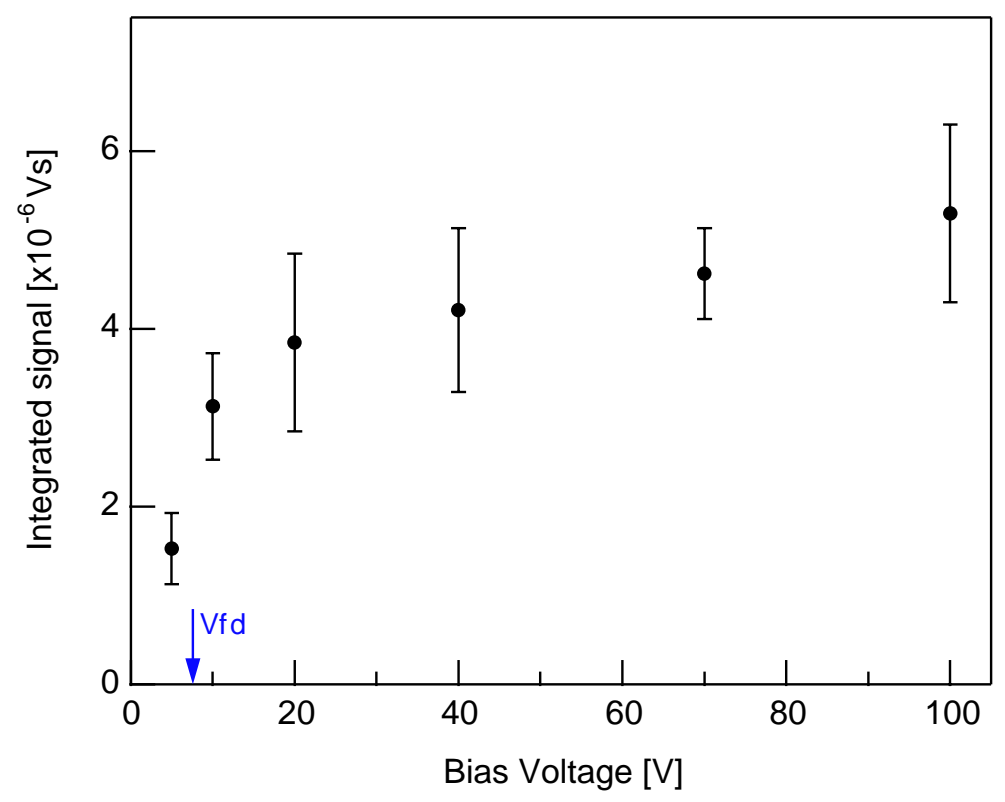

Fig. 5. Integrated signal registered on the central pad of the silicon beamcounter as a function of the bias voltage of the diode. Vfd indicates the voltage that is required to fully deplete the diode.

\subsection{The external beam detectors}

The external beam detectors monitor annihilation on the degraders (Fig.2). They consist of two types of modules, Front and Barrel, both made from $1 \mathrm{~cm}$ thick Bicron BC408 plastic scintillators [12]. The two Front detectors are $(195 \times 100) \mathrm{mm}^{2}$ each and the two Barrel detectors are $(800 \times 195) \mathrm{mm}^{2}$ each. The modules of 
the Front and Barrel detectors cover about $0.1 \%$ and $3 \%$ of $4 \pi$, respectively, when antiprotons annihilate on the degraders. Due to the high instantaneous rate $(\simeq$ $10^{14} \mathrm{~s}^{-1}$ ) during the $\bar{p}$ pulse, these detectors do not count single particles, but operate in current mode, measuring the total charge deposited by the annihilation products. However, the light produced in a $1 \mathrm{~cm}$ thick plastic scintillator would saturate the photomultiplier. We therefore used proximity focused Hybrid Photo Diodes (HPD) [13-15]. These comprise a vacuum tube in which the photoelectrons are accelerated toward a silicon diode by a high voltage applied to the photo-cathode. A gain of a few thousand can easily be achieved, low compared to photomultipliers $\left(\simeq 10^{7}\right)$ but sufficient for our application, in which a large amount of light is generated. We used two different models of HPD's built by DEP [16] namely model PP0350F for the Front and model PP0350D the for the Barrel detector. A detailed description of their characteristics can be found elsewhere [12].

In order to test the effect of magnetic field on HPD gain, we measured the pulse height produced by the HPDs in the ATHENA magnetic field using a pulsed light emitting diode (LED), held in the center of the photocathode. Measurements with and without magnetic field were consistent within $3 \%$, the difference being mainly due to LED instabilities.

\subsection{Absolute beam calibration}

An absolute calibration was performed by antiproton activation of an aluminium foil [17]. Antiprotons impinging on aluminium produce ${ }^{24} \mathrm{Na}^{*}$ (with a yield of 2.1 $\pm 0.3 \%$ ) which then decays by $\gamma$ emission with energies of $1369 \mathrm{keV}$ and 2754 $\mathrm{keV}$ [18]. The half life of ${ }^{24} \mathrm{Na}^{*}$ is $15 \mathrm{~h}$. We dumped the antiproton beam in an aluminum target (made of four foils with a thickness of $110 \mu \mathrm{m}$, glued together) located at the entrance of the ATHENA beam pipe. Using the induced activity, measured off-line with a calibrated germanium detector, an average beam intensity per shot of $(1.20 \pm 0.19) \times 10^{7} \bar{p}$ was obtained [12]. As an independent measure of beam intensity, the number of antiprotons in the $\mathrm{AD}$ ring was measured before extraction by a low noise Schottky pickup probe [19,20].

During the antiproton irradiation of the aluminium foil the annihilation signals were detected by the Front and Barrel external beam detectors and recorded with ADCs. Using the afore mentioned knowledge of beam intensity the Front and Barrel external beam detectors were calibrated as reported in Table 1. Here the measured ADC counts are converted to collected charge. While the Schottky probe gives the number of antiprotons inside the $\mathrm{AD}$ ring with high accuracy $(\simeq 1 \%)$, it is measured before electron cooling at $100 \mathrm{MeV} / \mathrm{c}$ and before extraction. Hence, possible cooling inefficiencies and losses in the ejection line might not be taken into account. However, the results shown in Table 1 are in excellent agreement with the activation method. 


\section{Table 1}

\begin{tabular}{|c|c|c|}
\hline Calibration Method & Front & Barrel \\
\hline Activation & $57.5 \pm 9.5$ & $27.2 \pm 4.5$ \\
\hline Schottky & $55.5 \pm 2.1$ & $26.1 \pm 1.0$ \\
\hline
\end{tabular}

Calibration factors of the external beam detectors measured by activation and with the Schottky probe (in $\mathrm{fC} / \bar{p}$ ), showing a good agreement between the activation and Schottky methods.

Fig. 6 shows the correlation between the charge measured with the silicon beam counter and the number of antiprotons determined with the external beam detectors. The former begins to lose linearity, due to saturation, starting at a flux of $\simeq 1 \times$ $10^{7} \bar{p} /$ pulse.

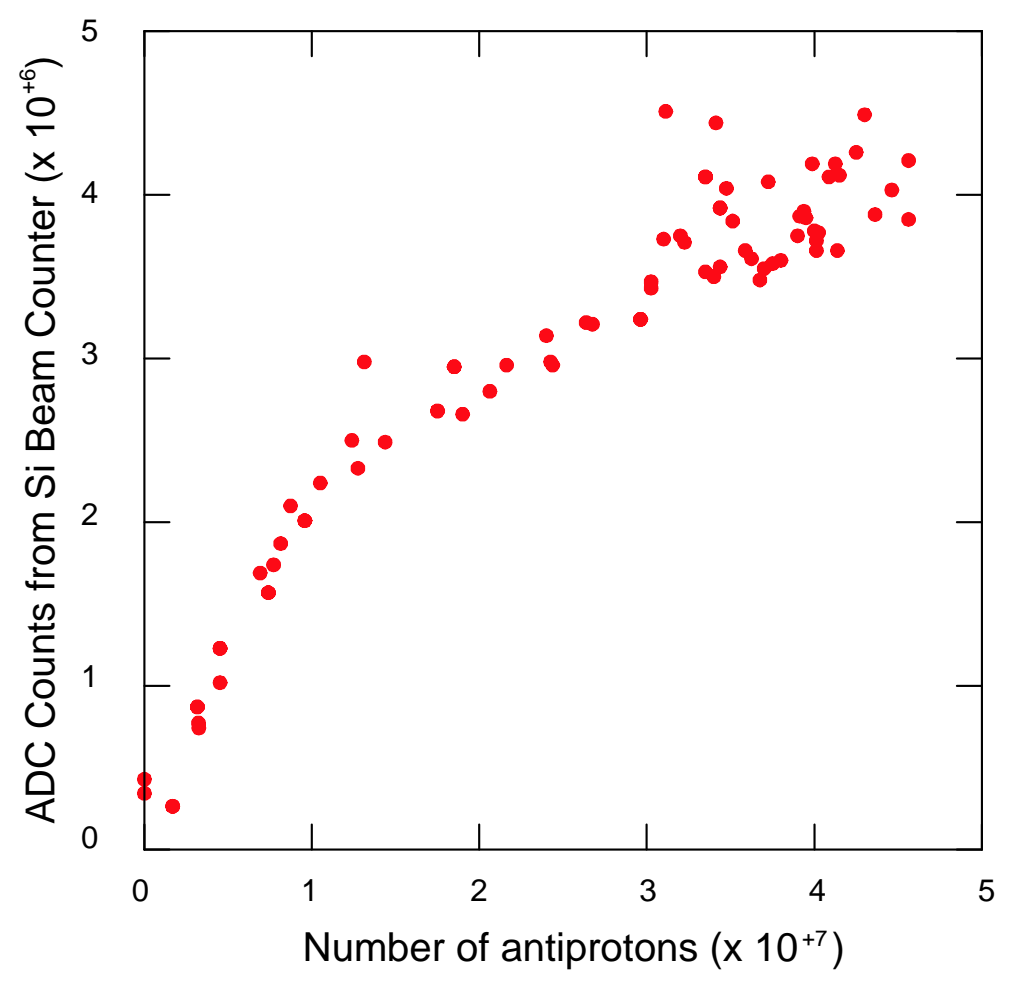

Fig. 6. Correlation between the number of antiprotons per AD pulse measured with the External Beam Detector and the charge collected by the silicon beam counter.

\subsection{The external annihilation detectors}

The solid angle coverage of the external annihilation detectors is sufficiently small (see below) to allow them to be used in single count mode, unlike the beam counters. This means they can be used to detect single $\bar{p}$ annihilations on the residual gas of the traps or on the trap walls. The external annihilation detectors consist of six coincidence pairs of Bicron BC408 plastic scintillators. Each scintillator is $10 \mathrm{~mm}$ thick, wrapped with aluminized mylar sheet, glued on one side to a fish tail shaped 
lucite light guide and read out by a Philips XP2020 photomultiplier. The horizontal scintillators are $(720 \times 300) \mathrm{mm}^{2}$ and the vertical scintillators $(600 \times 400)$ $\mathrm{mm}^{2}$, covering a solid angle of $\sim 30 \% \times 4 \pi$ with slight variations depending on the exact position of annihilation inside the traps. The photomultipliers are doubly screened from the magnetic field with a $\mu$-metal shield inside an iron housing and are mounted in a region where the stray magnetic field is small. They are biased to a negative voltage of $(2-2.5) \mathrm{kV}$, the gain being adjusted by changing the bias voltage, while the appropriate thresholds are determined using minimum ionizing particles, both from cosmic rays and from $\bar{p}$ annihilation. Pairwise coincidences allow operation at a lower threshold, reducing the random noise as well as suppressing neutral background from gammas and neutrons.

The discriminated signals are processed via NIM logic and are recorded with a VME multi-scalar module (Struck Instruments System, SIS3806), which is read out via a MXI bus onto a PC. The system allows dead-time free monitoring of trapped antiprotons with lifetimes longer than several hours [21]. Typical background rates are $\sim 20 \mathrm{~Hz}$ per coincidence pair, but can be reduced by further requiring charged particle multiplicities larger than or equal to two. The signals can also be used to trigger the antihydrogen detector readout (see section 8).

The excellent correlation between the beam intensity measured by the external beam detector and the number of annihilations of trapped antiprotons measured by the external annihilation detector is illustrated in Fig. 7. The details of antiproton trapping and cooling are discussed in section 4.1.

\section{The trap system}

The trap system is realized by a sequence of electrodes having $1.25 \mathrm{~cm}$ inner radius and various lengths. Different sections of this system are used to perform different functions. Proceeding from the antiproton beam entrance and moving toward the positron accumulator, the first 12 electrodes are used to catch, cool and accumulate antiprotons (catching trap). The following group of electrodes is referred to as the mixing trap. This is the region where the antiprotons are merged with the positron plasma. The last group of electrodes is used during the positron transfer and recapture procedure.

All the electrodes outside the mixing region are copper, plated with gold to avoid oxidation. Below, a 0.5-1 $\mu \mathrm{m}$ thick palladium sub-layer was deposited to improve plating performance and avoid the diffusion of gold. Static or varying voltages can be applied independently to each electrode allowing the electric field inside the trap to be shaped according to the particular operation required. 


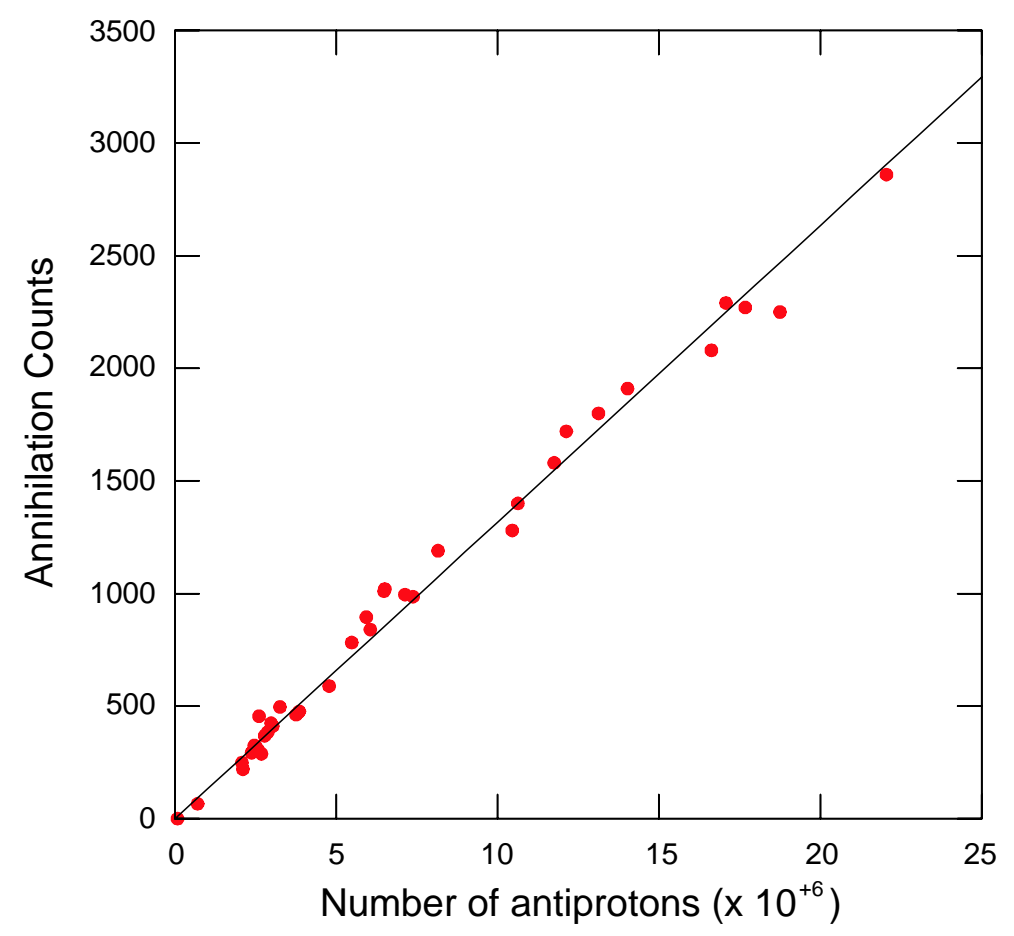

Fig. 7. Counts measured by the external annihilation detectors upon the release of trapped antiprotons as a function of antiproton number in the incoming beam as measured by the external beam detectors.

\subsection{The catching trap}

The catching trap is composed of 12 cylindrical electrodes. (Fig. 8). The two outermost electrodes (HVL and HVR) supply the high voltage to catch the incoming AD antiproton bunches. The central part of the trap, comprised of 10 electrodes, is used to confine the cold antiprotons. This section includes seven electrodes designed to produce an harmonic potential (Penning trap) along the axis by an appropiate choice of the electrode lengths and applied voltages [22]. One of the harmonic region electrodes was split in four in order to allow the application of the rotating wall technique for plasma compression [23] or, alternatively, to detect plasma diocotron modes [24].

\subsection{The mixing trap}

The mixing trap is the region where the interaction between antiprotons and positrons takes place (see fig. 9). The electrodes have been arranged in such a way that the "nested" configuration [25] can be achieved. In this configuration particles of opposite charge can be confined simultaneously and made to interact. During the mixing of the two clouds recombination can take place. In essence the mixing region consists of three sections marked $\mathrm{RW}, e^{+} \mathrm{W}$ and $\mathrm{LW}$ in fig. 9; in each of them it is 


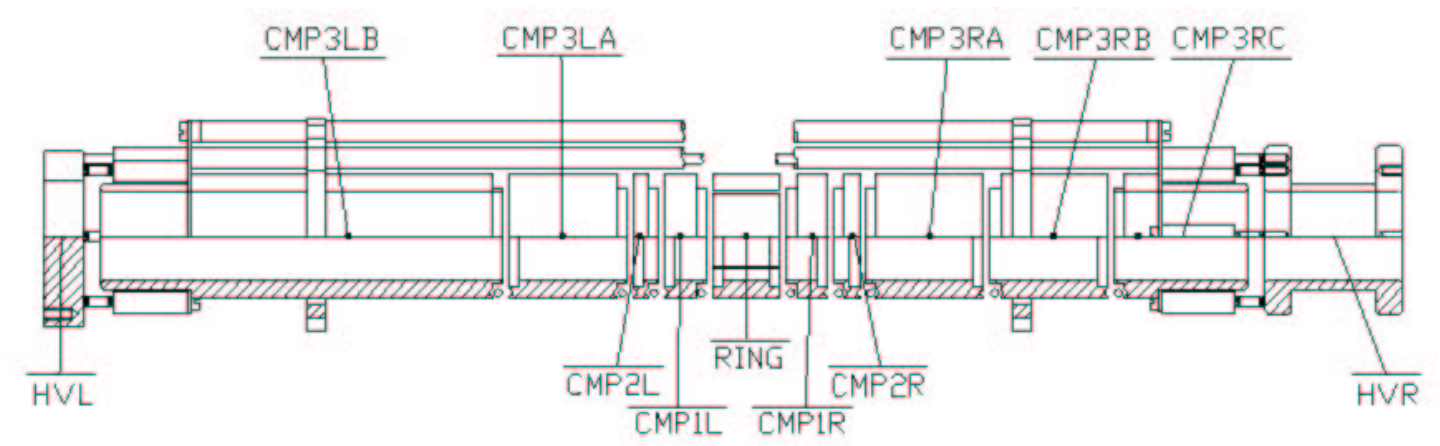

Fig. 8. The antiproton catching trap. The trap design allows the application of up to $15 \mathrm{kV}$ to the two outermost electrodes. The central section is a "7 electrode design"; characterized by the presence of a central electrode (RING) and by 3 compensation electrodes on each side. The central electrode is a 4-way split in order to drive and detect azimuthal plasma modes.

possible to set up a harmonic potential well as described for the catching trap. The positrons are normally confined in the central of these three traps $\left(e^{+} \mathbf{W}\right)$. All the electrodes of the mixing region are $3.25 \mathrm{~mm}$ thick and are made of aluminum as they are located in the antihydrogen production zone inside the annihilation detector. The use of aluminum instead of copper, combined with the thinner wall thickness, makes it possible to significantly reduce multiple scattering of the annihilation products on their passage to the detector, as well as avoid conversion of high energy photons to electron-positron pairs, which if they occured inside the detector volume would lead to an increased background. Outside the mixing zone, the electrodes are $4 \mathrm{~mm}$ thick. As in the catching trap, one of the electrodes in the positron trap section has been split in four azimuthal quadrants to allow for radial compression using the rotating wall technique.

\subsection{Additional electrodes}

To increase the experimental flexibility of the traps a section made up of nine electrodes and approximately $21 \mathrm{~cm}$ in total length was added on the side of the mixing trap towards the positron accumulator (R1-9 in fig. 9). These electrodes are used during the transfer of positrons to the mixing trap and can act as additional particle reservoirs for positrons or electrons, thus perhaps in the future allowing transfer and stacking of positrons during the recombination phase. At the end of the mixing trap a high voltage electrode was added (HVRR in fig. 9); it can be biased to a potential of about $5 \mathrm{kV}$. In this way, antiprotons can be trapped directly in the mixing section and cooled by either electrons or perhaps directly by a positron cloud produced previously by stacking several accumulation cycles of positrons (see section 7). In this way high densities and particle numbers of positrons could be utilised to further optimise the antihydrogen formation process. 


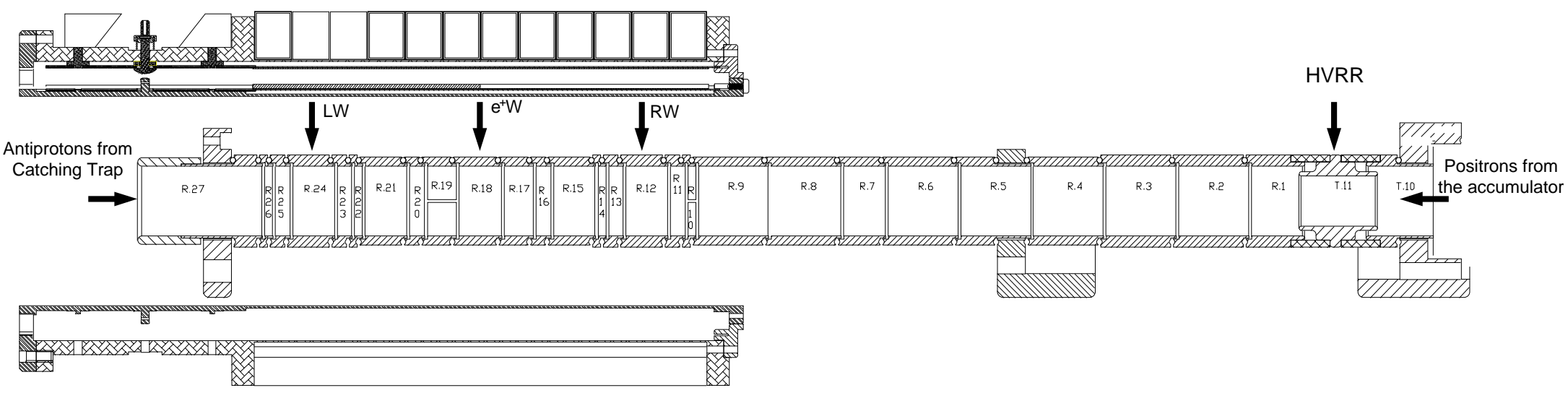

Fig. 9. The mixing trap. Antiprotons from the catching trap arrive at the left and positrons from the positron accumulator (section 7) arrive at the right. The "nested" trap is made up of 3 sections each with the possibility of making a harmonic well. The two outer ones of these (RW and LW) are intended for the antiprotons and the central larger trap $\left(e^{+} \mathrm{W}\right)$ is intended for the positrons. The position of the antihydrogen detector (section 8$)$ is shown. The additional high voltage electrode (HVRR) is marked. 


\section{Antiproton capture, cooling and manipulation}

The standard operating procedure for antiprotons in ATHENA consists of their capture in the catching trap, cooling them by collisions with a preloaded cloud of electrons and transferring the cold antiprotons to the mixing trap. The last aluminum degrading foil is mounted on the trap entrance electrode HVL. The potential of the exit electrode (HVR) at the other end of the catching trap is initially set to $V_{H V}$. Antiprotons traversing the foil with axial energy lower than $e V_{H V}$ are reflected from HVR and captured by applying a voltage $V_{H V}$ to HVL before they return there. The antiproton arrival time is determined by the silicon beam counter (see section 3.1). The optimum trap closing time, which depends on bunch duration and energy and on the trap length, was determined experimentally.

The number of captured antiprotons is determined by the external annihilation detectors (see section 3.4) by lowering the voltage of the entrance electrode (with a $5 \mathrm{~ms}$ time constant), thus allowing the antiprotons to annihilate on the degrader. The time distribution from the detector is related to the total number of captured antiprotons and their axial energy distribution. Figure 10 shows how the number of captured antiprotons increases with applied trap potential. Typically around 10000 antiprotons are captured at $5 \mathrm{kV}$ for an incident AD flux of $2.5 \times 10^{7} /$ pulse. Figure 11 shows how the number of captured antiprotons decreases when the trap closing time is increased. The optimum closing time is 500-700 ns. Note the slow decrease in catching efficiency as a function of closing time, indicating that a significant fraction of the antiprotons leave the degrader with very low energy.

\subsection{Antiproton cooling}

Coulomb collisions between antiprotons and electrons preloaded in the catching trap can efficiently cool the high energy antiprotons [26]. Although the electrons are heated by this process, they cool themselves by synchrotron radiation in the 3 Tesla magnetic field with a time constant of about $0.4 \mathrm{sec}$. Ideally, the two species of particles will reach a final equilibrium temperature equal to that of the environment. The cooling process is usually described by the differential equations [27]

$$
\begin{gathered}
\frac{d T_{p}}{d t}=-\frac{\left(T_{p}-T_{e}\right)}{\tau_{c}} \\
\frac{d T_{e}}{d t}=\frac{n_{p}}{n_{e}} \frac{1}{\tau_{c}}\left(T_{p}-T_{e}\right)-\frac{\left(T_{e}-T_{t}\right)}{\tau_{e}},
\end{gathered}
$$

where $T_{e}$ and $T_{p}$ are the electron and $\bar{p}$ temperatures, $T_{t}$ is the unperturbated electron temperature, $n_{e}$ and $n_{p}$ are the electron and $\bar{p}$ densities, $\tau_{e}$ is the synchrotron and $\tau_{c}$ the electron cooling time. The latter is given by 


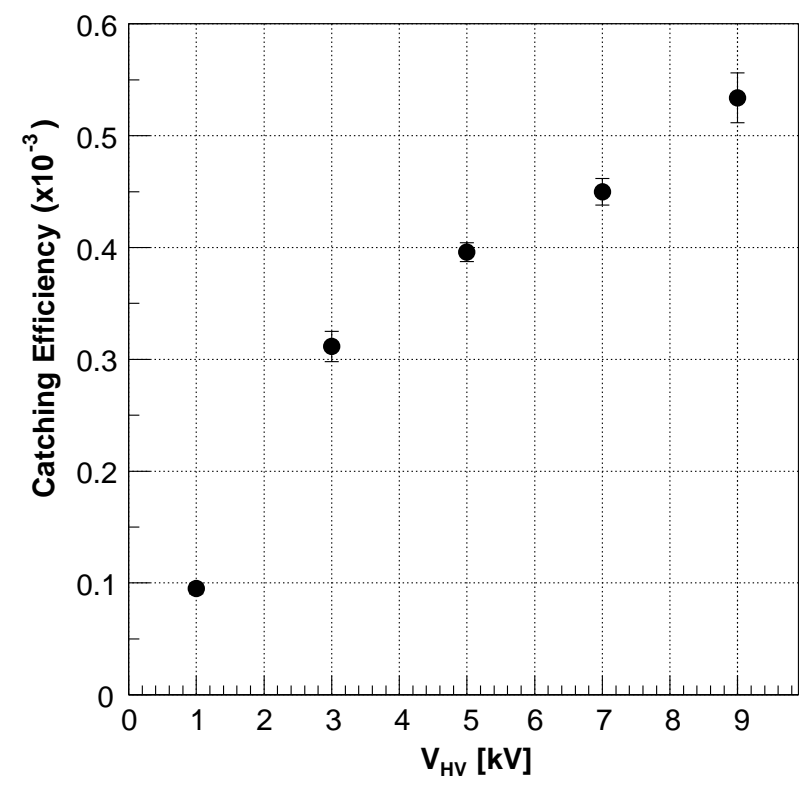

Fig. 10. Dependence of the catching efficiency on the applied high voltage, $V_{\mathrm{HV}}$. The antiprotons were released from the trap $1 \mathrm{sec}$ after capture. The numbers of captured antiprotons are normalized to the beam intensity measured with HPD-based external beam detectors.

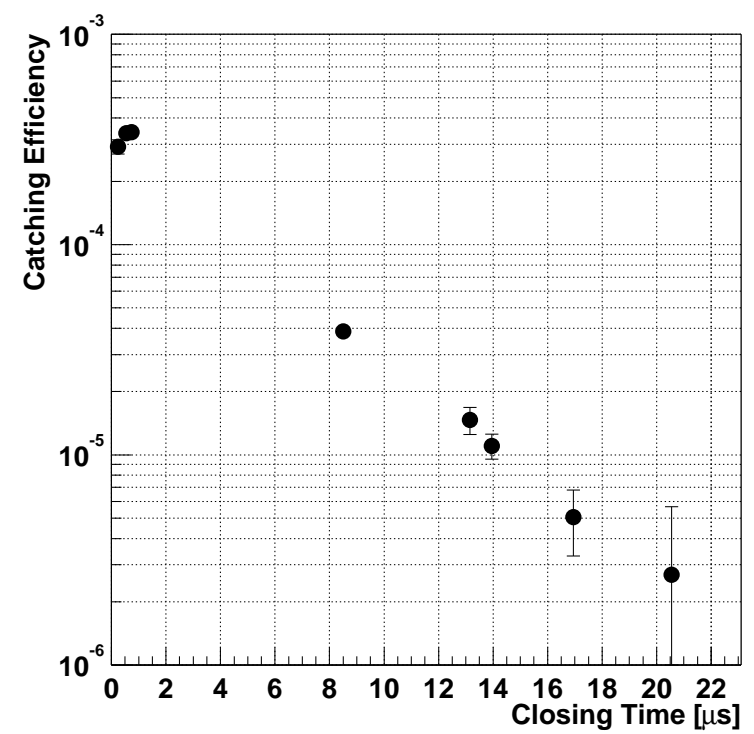

Fig. 11. Dependence of the catching efficiency on the trap closing time delay.

$$
\tau_{c}=\frac{3 m_{e} m_{p} c^{3}}{8(2 \pi)^{1 / 2} n_{e} e^{4} \ln (\Lambda)}\left(\frac{k T_{p}}{m_{p} c^{2}}+\frac{k T_{e}}{m_{e} c^{2}}\right)^{3 / 2} .
$$

Here $m_{p}$ and $m_{e}$ are the $\bar{p}$ and electron masses, $e$ is their electrical charge and $\Lambda$ is given by

$$
\Lambda=\frac{4 \pi \epsilon_{0}}{n_{e}}\left(\frac{k T}{e^{2}}\right)^{3 / 2} .
$$


The solution of these equations shows that $10^{4}$ antiprotons having energies in the $\mathrm{keV}$ range can be cooled down to less than a few $\mathrm{eV}$ within a few tenths of a second if they overlap completely with an electron cloud of density around $10^{7}-10^{8} \mathrm{~cm}^{-3}$.

The ATHENA electron source consists of a barium oxide disc cathode (Kimball Physics Inc.) mounted on a movable support in the positron transfer region (section 7.3). The electrons are loaded in the $\bar{p}$ catching trap before the arrival of the antiproton pulse. This is achieved by shaping a narrow, low-voltage potential well (typically a few tens of volts) in the central region of the catching trap. The electron primary current traverses the catching trap and reaches the entrance electrode HVL where it may be dumped on the degrader foil or repelled by it depending on the electric field configuration. Electrons can be loaded in different sections of the catching trap by various procedures. They differ from each other by the values of the voltages applied to the trap electrodes during the primary current passage and they lead to clouds having different initial shapes and densities and distinct evolution dynamics.

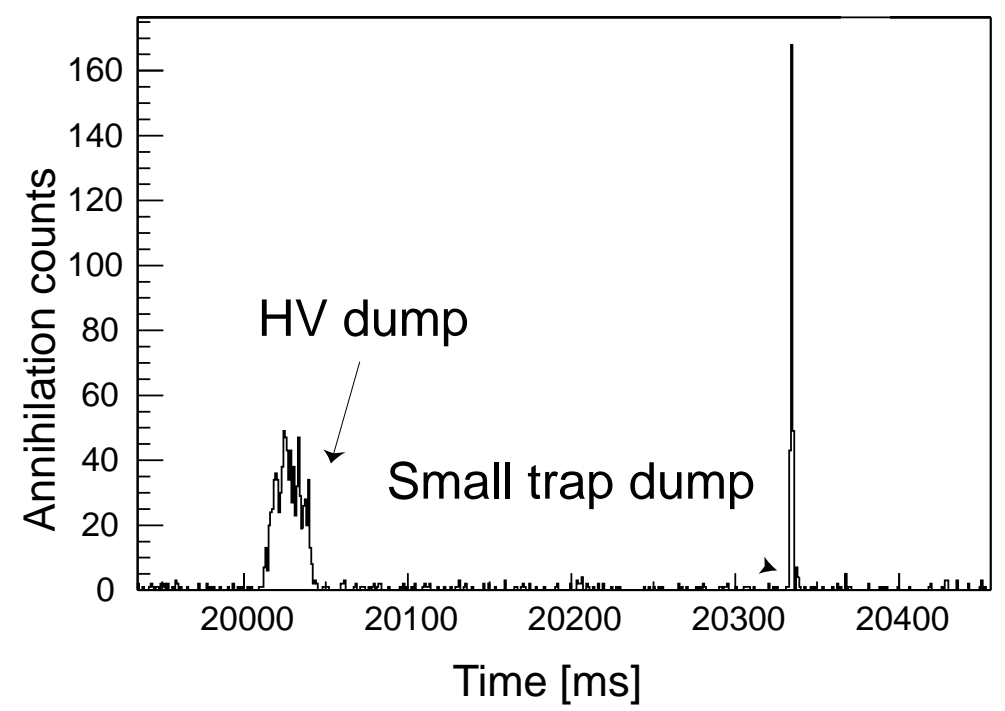

Fig. 12. A typical $\bar{p}$ annihilation time spectrum measured by the external annihilation detector (see text). The clock is started at injection of the $\bar{p}$ beam into the trap.

The cooling process is studied by dumping the "hot" antiprotons from the high voltage well and then later the "cold" antiprotons cooled by the electrons and captured in the narrow internal electron well. Figure 12 shows a typical time distribution taken with the external annihilation detectors for trapped antiprotons released and annihilating on the degrader at the entrance of the trap. The electric potential was first lowered from $5 \mathrm{kV}$ to $40 \mathrm{~V}$ (with a time constant of $\sim 20 \mathrm{~ms}$ ) releasing the higher energy antiprotons (HV dump in the figure), and then from $40 \mathrm{~V}$ to $0 \mathrm{~V}$ in about $1 \mathrm{~ms}$, thus releasing the antiprotons which had been cooled via interaction with preloaded electrons (small trap dump). Figure 13 shows the fraction of cold and hot antiprotons as a function of interaction time. Nearly all antiprotons are cooled in about $60 \mathrm{~s}$. At the end of the cooling process, antiprotons and electrons 
share the same volume. The electrons can then be ejected from the trap by applying appropriate electric pulses of about 100 ns duration which do not affect the heavier antiprotons.

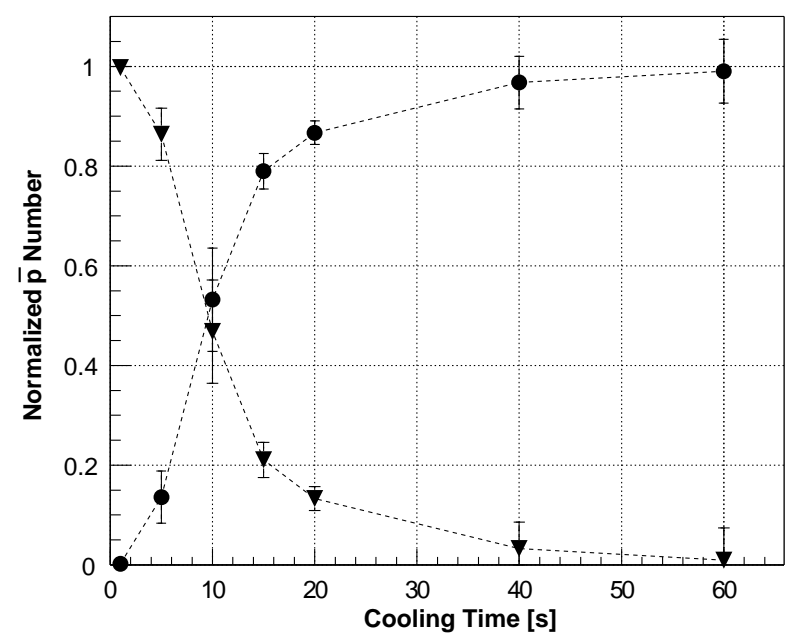

Fig. 13. Measured fraction of cold (circles) and hot antiprotons (triangles) as a function of their interaction time with electrons. The dashed lines are to guide the eye.

Since the catching process does not influence the potentials within the central region where the cold antiprotons and the electrons are collected, several AD shots can be stacked in the catching trap. This is illustrated in Fig. 14 which shows a linear increase in the number of trapped cold antiprotons with the number of AD shots.

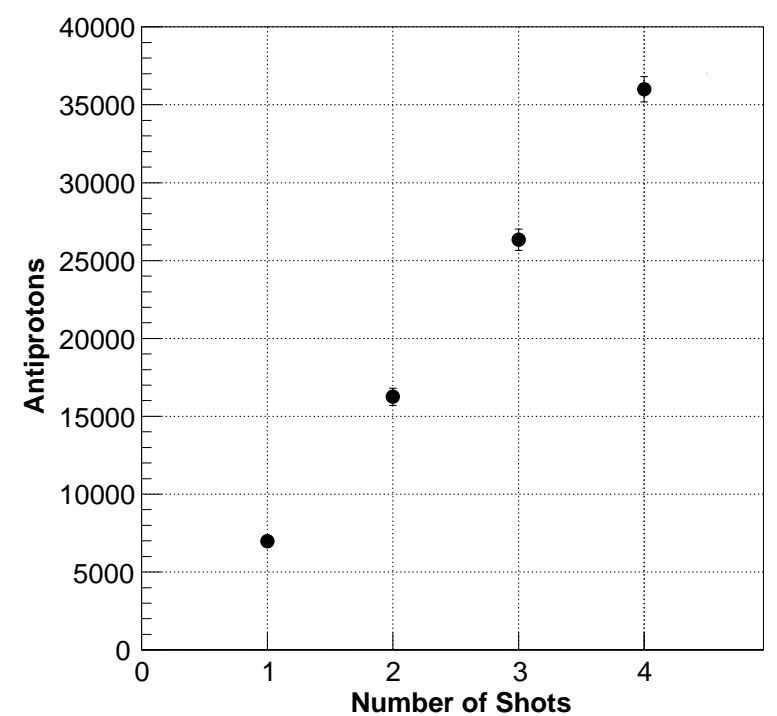

Fig. 14. Dependence of the number of cold antiprotons on the number of stacked AD shots. Each AD shots contains about $2 \times 10^{7}$ antiprotons. 


\subsection{Electron plasma characteristics}

The high density and low temperature of the electron cloud makes the Debye length shorter than the trap cloud extension and means that the electron cloud is well within the plasma regime. The evolution of the electron plasma can be studied using destructive or non-destructive diagnostic systems. The destructive diagnostic simply consists of dumping the electron cloud onto the degrader mounted on HVL (see figure 8), which also acts as a Faraday cup, and reading the collected charge by means of a low-noise high-impedance amplifier. A non-destructive diagnostic based on monitoring of the axial plasma modes is also implemented, and will be discussed in Section 6.

The electron storage time is limited by collisions with the residual gas, asymmetries and imperfections in the trap geometry and in the fields, leading to radial transport across the magnetic field and expansion of the plasma. This is a well documented phenomenon in the field of nonneutral plasmas [28]. In ATHENA, electron plasmas of few $\mathrm{cm}$ in length with several $10^{8}$ electrons are routinely loaded in the catching trap. The electron storage time depends on the trap length, the depth of the potential well and the choice of electrodes. Typical electron storage times (the time necessary for the particle number to reduce by a factor two ) range from a few hundred to a few thousand seconds $[29,30]$.

\subsection{Antiproton storage time}

Figure 15 shows the number of cold antiprotons (i.e. captured in the narrow well) as a function of time, in the presence of electrons or after having ejected them. The background pressure as measured in the room temperature region of the apparatus external to the cryogenic region (see section 2.2) was about $10^{-11}$ mbar and the trap temperature $15 \mathrm{~K}$. We observed that the electrons reduce the cold antiproton storage time; without electrons the half-life of the antiproton cloud is usually better than 10 hours.

\subsection{Antiproton transfer}

Cold antiprotons are transferred toward the mixing region by adiabatically moving the electrode voltages along the traps. The transfer efficiency in the case when electrons and antiprotons are moved together are compared with those when electrons are first ejected from the catching trap and only the cold antiprotons are transferred. Transfer efficiencies greater than $90 \%$ are obtained when electrons and antiprotons are transferred together while a dramatic decrease in the transfer efficiency has been observed if cold antiprotons are moved alone. In addition, while the number 


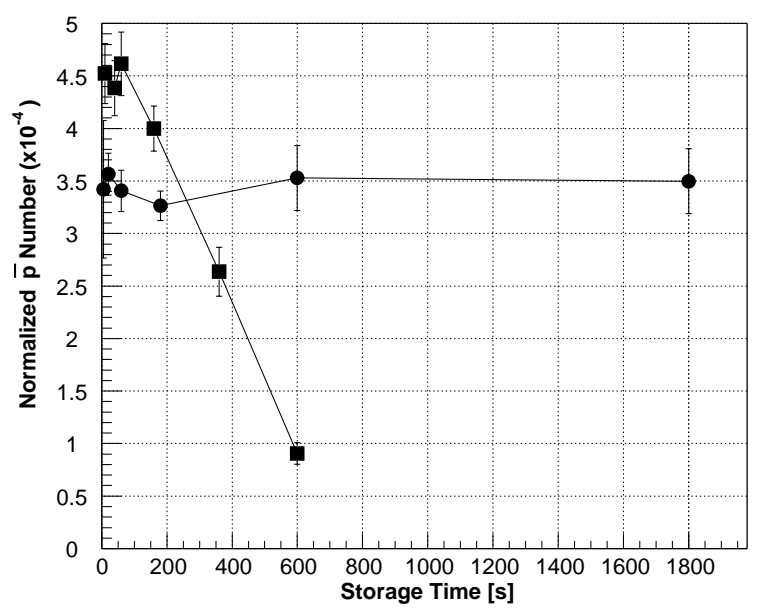

Fig. 15. Storage time for cold antiprotons with (squares) and without (circles) electrons. The antiproton numbers are normalized to the beam intensity measured with the HPD-based external beam detectors. The lines are to guide the eye.

of cold antiprotons stored in the catching trap linearly increases with the number of AD shots (Fig. 14), under the experimental conditions of 2002 the transfer efficiency did not follow the same behaviour. Thus when stacking several shots in the catching trap prior to transfer, most of the transfered antiprotons (about 75\%) originated from the last trapped bunch. This behaviour was unexpected and could be related to possible radial (centrifugal) [31] separation of antiprotons and electrons leading to instabilities during the transfer. An improvement in the electron loading and especially the antiproton transfer procedure during the ongoing 2003 run has solved this problem. The normal procedure in the antihydrogen production runs in 2002 required the stacking and transferring of 3 AD shots resulting in about 10000 cold antiprotons available for recombination in the mixing trap.

\section{Plasma modes diagnostic}

The thermal equilibrium state of a large number of positrons or electrons confined in a Penning trap at low temperature is a rigidly rotating spheroidal plasma [32] with a sharp boundary. Models predict that the density is almost constant within the ellipsoid and that it falls off exponentially with the Debye length at the plasma boundary.

Knowledge of the characteristics of the plasma (i.e. dimensions, density, temperature) can be obtained by means of a non-destructive method based on measurement of the first two axial electrostatic mode frequencies (dipole, quadrupole) [33,34].

The modes are excited by applying sinusoidally time-varying potentials to one trap electrode, while the plasma response can be measured by acquiring the induced 


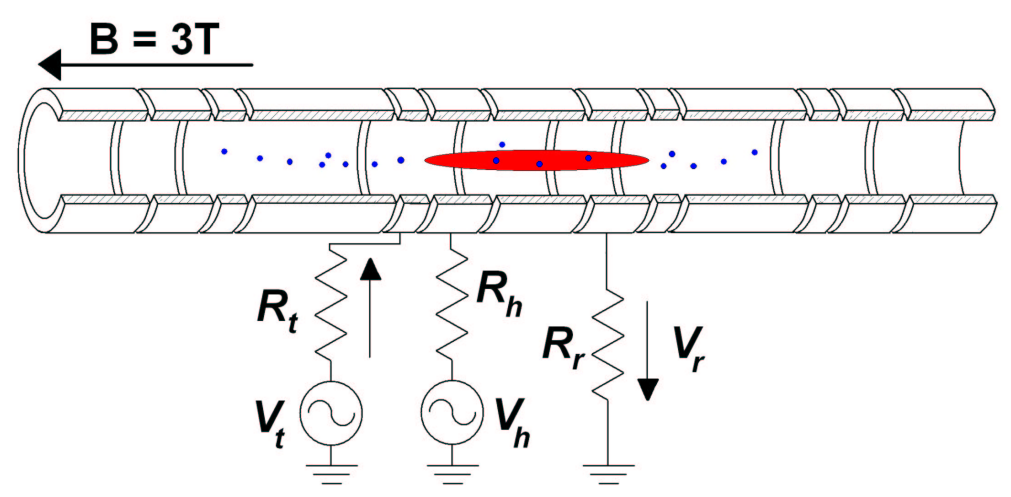

Fig. 16. Schematic diagram of a Penning trap with the modes analysis and heating circuit

current on another electrode (see figure 16). The ratio of the induced current to the excitation amplitude is measured as a function of the drive frequency. A narrow step-wise frequency sweep ( $4 \mathrm{~ms}$ duration per $5 \mathrm{kHz}$ step) is made across the resonant frequency of each mode. For each frequency step, the amplitude and phase (relative to that of the drive signal) of the voltage induced by the plasma motion is acquired. This excite-detect process is performed by means of a network analyzer (HP4395) integrated with suitable attenuation and amplifying circuits. The cross talk signal between the transmitting and receiving electrodes is acquired without positrons and subtracted from the signal measured with the plasma present.

A detailed and simple analytic theory of the electrostatic modes in nonneutral plasmas exists [33]. In the framework of this theory the frequencies of the first two symmetric axial modes depend on the plasma size, density, and temperature. The zero temperature model is used to determine the plasma density and the so called aspect ratio (which is the ratio between the axial and radial extension of the plasma).

Moreover the exact plasma response (see Fig.17) can be modelled using a resonant circuit analogy, where the values of the components are related to the plasma properties. The diagnostic also allows, in addition to the the previous parameters, the plasma length $\left(2 z_{0}\right)$ to be obtained. The radius $\left(r_{0}\right)$ and the particle number $(N)$ are then determined and a complete nondestructive diagnostic system is obtained. Temperature $(T)$ shifts produced by the application of a radiofrequency signal resonant on the $(1,0)$ mode are monitored as changes in the $(2,0)$ mode frequency. The model [35] is used to calculate the induced temperature increase. The diagnostic system as well as the temperature monitoring and control is described in more detail elsewhere [36,37]. 

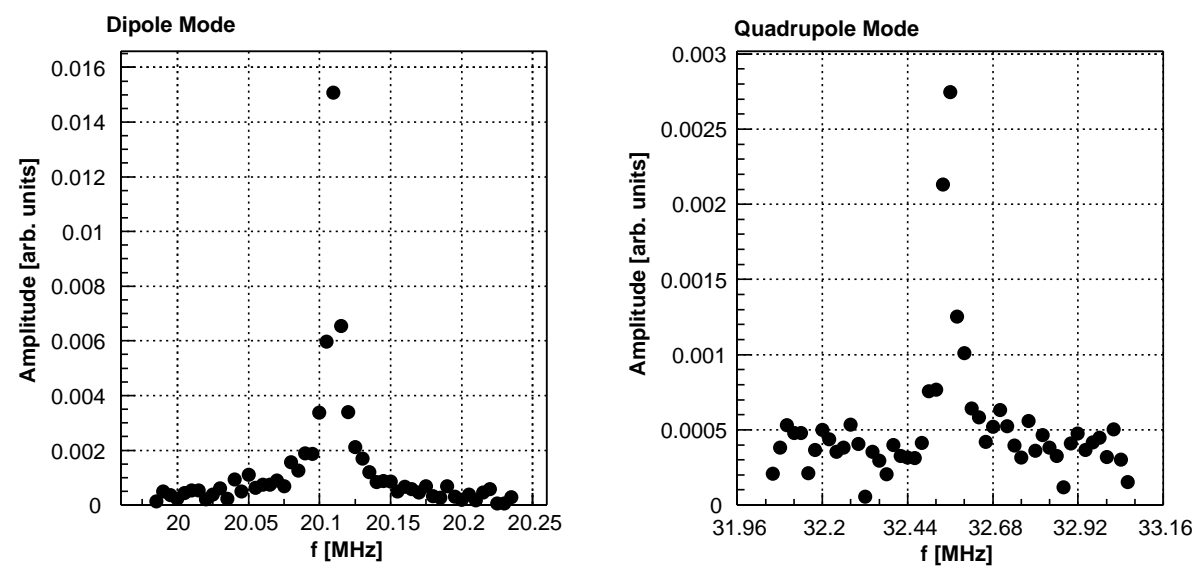

Fig. 17. Measurement of the amplitude of the first two low-order axial modes as a function of the drive frequency. In this case the measured plasma parameters are $n=6.3 \cdot 10^{7} \mathrm{~cm}^{-3}$, $z_{0}=2.0 \mathrm{~cm}$, and $r_{0}=0.1 \mathrm{~cm}$.

\section{Positron accumulator}

\subsection{Overview and operation}

The operation of the ATHENA positron accumulator is based on the buffer gas capture and cooling of positrons in a Penning-Malmberg trap. The techniques used were pioneered by the University of California San Diego positron [38-40] and electron [23,41,42] groups. As described below, up to $2 \times 10^{8}$ positrons have been trapped in this accumulator prior to transferring them across a low field region to the main ATHENA recombination trap.

The accumulator traps and cools a continuous beam of slow positrons. These are generated by moderating $\beta^{+}$particles from a $1.4 \mathrm{GBq}(40 \mathrm{mCi}){ }^{22} \mathrm{Na}$ radioactive source and guiding them into the trapping region using axial magnetic field transport. A cryogenic cold head capable of reaching $5.5 \mathrm{~K}$ cools down the source and makes it possible to grow a solid neon moderator directly on the source [4345]. Details of the design of the source holder and its interface with the cryogenic section can be found elsewhere [46-48]. Figure 18 shows the trapping region. A $\mathrm{NaI}(\mathrm{Tl})$ detector is located close to a gate valve which can be used to isolate the source/moderator end of the apparatus and the main trapping region. This facilitates optimisation of moderator growth, with the closed valve used as a simple positron annihilation target. The absolute beam intensity was obtained by crosscalibrating the $\mathrm{NaI}(\mathrm{Tl})$ detector and performing coincidence measurements with a channeltron detector which was periodically placed in the beam-line. Moderation efficiencies (absolute beam intensity divided by the total positron activity of the source) of around $0.4 \%$ are routinely achieved such that beam intensities greater than $5 \times 10^{6}$ positrons per second are available. 


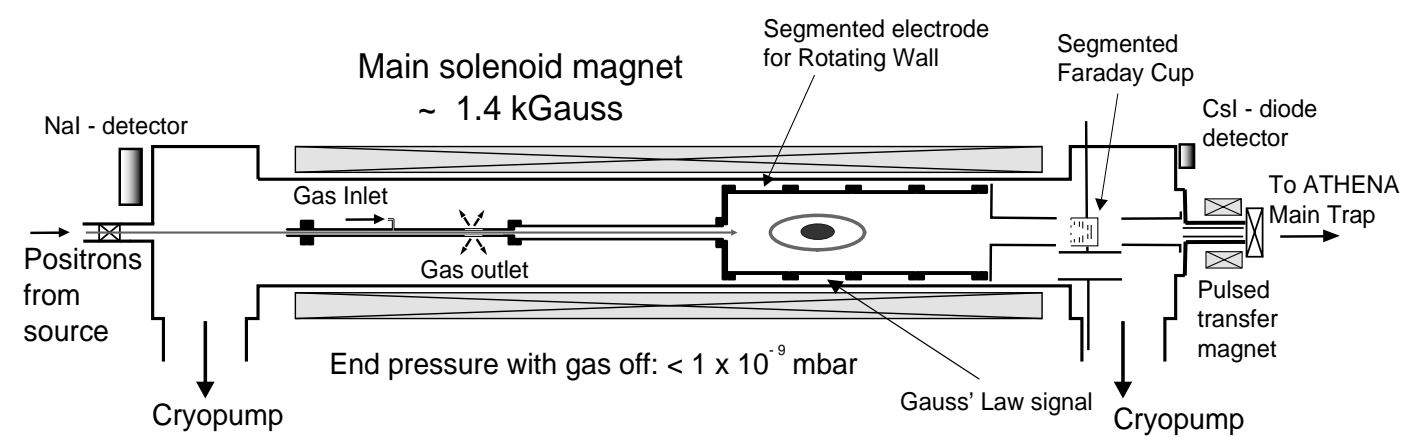

Fig. 18. Schematic overview of the positron accumulator (see text).

The trapping scheme utilizes nitrogen buffer gas to trap and cool the positrons. Initial trapping occurs during the first passage of the positron through the trap electrodes by electronic excitation of the nitrogen gas. Such a transition is favoured in nitrogen compared to positronium formation, which is the only other major inelastic channel open at our kinetic energies. After trapping, axial confinement is provided by applying appropriate electric potentials to the electrode array, whilst the radial confinement is provided by a $0.14 \mathrm{~T}$ axial magnetic field. Once trapped the positrons continue to lose energy in collisions with the gas, finally residing in the potential well formed by the voltages applied to the large diameter trap electrodes.

One of the trapping electrodes (see fig. 18) is split into six segments to compress the plasma by applying a rotating electric field (the so-called "rotating wall" technique) $[23,42]$. In this technique the rotating electric field transfers torque to the plasma resulting in radial compression. The method was recently shown to work well for positron plasmas [49,50]. In the present apparatus the electrodes used have a significantly larger radius $(\simeq 10 \mathrm{~cm})$ than in these earlier experiments, but results presented below show that the positron plasma can still be influenced. Rotating wall compression leads to heating of the plasma, and since the magnetic field in the trap is too low for efficient re-cooling by synchrotron radiation, another cooling mechanism has to be used. We have successfully used the nitrogen buffer gas already present in the trap to provide this cooling, despite the fact that this gas has a poor positron cooling rate $[39,51,52]$. The presence of the segmented electrodes in the accumulation trap allow rotating wall compression during positron accumulation. This reduces positron losses due to cross-field transport in the presence of the buffer gas, leading to a larger number of accumulated positrons.

The detection system used to monitor the performance of the positron accumulator has two main components: (i) a segmented Faraday cup detector consisting of nine plates to extract information on the size and position of the plasma. The Faraday cup has an area of $25 \mathrm{~cm}^{2}$ and it is situated outside the main magnet in a region where the magnetic field is about a quarter of that inside the trap. This means that the plasma size obtained directly from the Faraday cup is magnified compared to its actual size inside the magnet and this has been taken into account to derive the 
true plasma dimensions; (ii) a calibrated CsI-photodiode detector (see section 7.4) to monitor the annihilation signal generated when the positrons strike the Faraday cup.

After trapping, the positrons still need to be transferred to the main magnet containing the mixing trap. A transfer section was constructed for this purpose. This section consists of a vacuum separation valve, a pumping restriction, a number of transfer electrodes and a transfer magnet capable of pulsing from 0 to $1 \mathrm{~T}$ in $20 \mathrm{~ms}$ and staying at $1 \mathrm{~T}$ for $1 \mathrm{~s}$. The performance of this system is reported below.

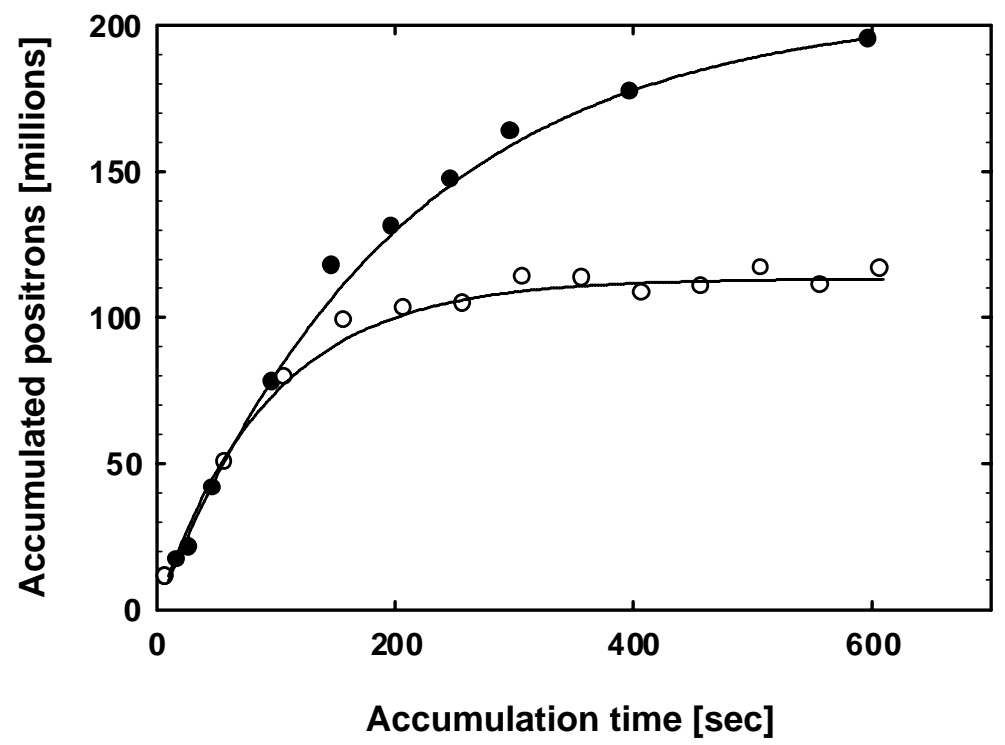

Fig. 19. Accumulation of positrons with (closed circles) and without (open circles) rotating wall compression. The lines are fits to the data (see text for details)

\subsection{Optimisation and results}

An optimisation programme was undertaken to tune the performance of the accumulator. We varied the electrode potentials and buffer gas pressures and fine-tuned the alignment of the magnetic field to the physical axis of the system. Figure 19 shows the end result, the accumulation of more than $10^{8}$ positrons in a few minutes. When using a suitable frequency and amplitude for the rotating wall compression (see below in this section) and applying this signal for the last $50 \%$ of the accumulation time, the lifetime of the positrons can be doubled in the presence of the buffer gas whilst maintaining the same accumulation rate. The data using the rotating wall compression (fig. 19) was fitted with a lifetime of $200 \mathrm{~s}$ whilst the data without the rotating wall gave a lifetime of $95 \mathrm{~s}$. It is important to stress that these results occur with the buffer gas still present in the trap. The increase in lifetime with rotating wall compression shows that annihilation on the gas is not the dominant loss. This points instead to plasma loss due to collision-induced cross-field drift to the electrodes. 
Plasma compression was optimised by mapping out the properties of the plasma as a function of the frequency and amplitude of the applied rotating electric field. The CsI-photodiode detector was used to record the total number of stored positrons, whilst the segmented Faraday cup monitored their position. Plasma centering could then be observed by the signal increase on the central plates of the Faraday cup. The ratios between the signals on the various Faraday cup plates yielded positional information and absolute plasma sizes.
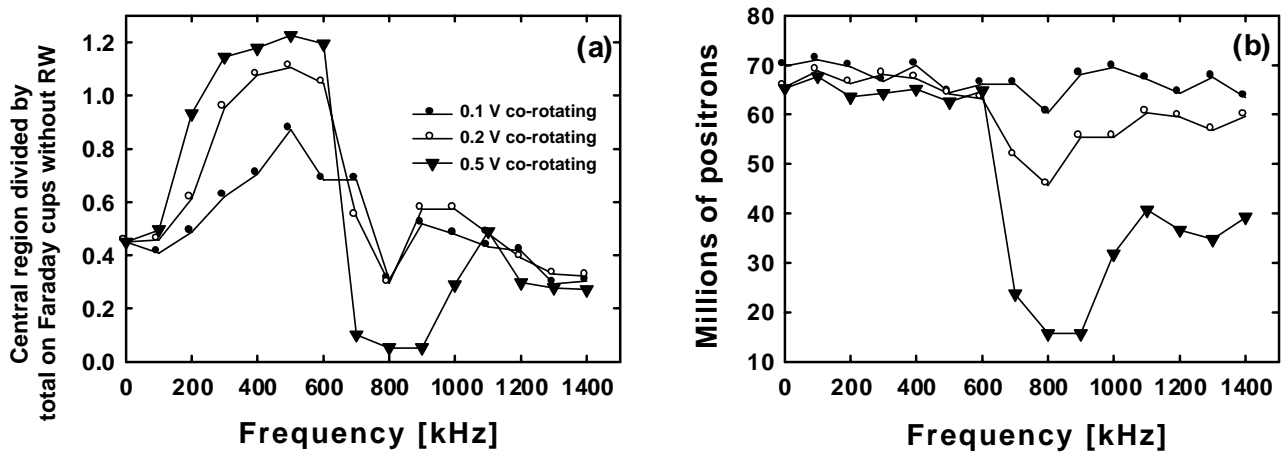

Fig. 20. (a) ratio of positron numbers in the central region of the Faraday cup to the total signal with and without rotating wall compression; (b) corresponding signal from the CsI-photodiode detector.

Figure 20 (a) shows measured ratios between signals from the central region of the Faraday cup with and without rotating wall compression. The central region covers about $20 \%$ of the total area. The direction of rotation of the applied electric field coincides with that of the natural $(\vec{E} \times \vec{B})$ rotation of the plasma. Figure 20 (b) shows the corresponding total number of positrons observed by the CsI photodiode detector. The data in fig. 20 (a) exhibit a broad enhancement in the compression in the frequency range $300-600 \mathrm{kHz}$ increasing with amplitude. The fact that the ratio for the central region rises above unity means that parts of the positron plasma which initially missed the Faraday cup, e.g. due to cross-field transport, have been compressed into the central region. Above $600 \mathrm{kHz}$ an abrupt fall-off occurs which coincides with a similar decrease in the total number of stored positrons. Up to 600 $\mathrm{kHz}$ the total number of positrons is very stable for all amplitudes.

The data in fig. 20 indicate a compression of about 2.5. However, the true compression turns out to be larger. The central region of the Faraday cups contained five individual plates, of which only three actually recorded a signal when the rotating wall was used. By examining e.g. ratios between adjacent plates and comparing to similar data when the Faraday cup was moved slightly off axis, it was possible to derive the position and size of the plasma. The plasma had a width of 15 $\mathrm{mm}$ (FWHM) when no rotating wall was applied, reduced to 3-4 $\mathrm{mm}$ following compression. Since the total number of positrons stayed constant, or in some cases increased, the central density increased by more than a factor of ten. This compression ratio is much larger than that reported previously for $\mathrm{N}_{2}$ [50]. This can possibly be attributed to the higher gas pressures used in the present study. 


\subsection{Positron transfer}

After accumulation the positrons are transferred to the mixing trap inside the main $3 \mathrm{~T}$ magnet. The nitrogen buffer gas is pumped out and, after the pressure in the positron accumulator has fallen below $10^{-8}$ mbar, the valve is opened and a pulsed transfer magnet is energised for 1 second. This transfer magnet produces a field of $1 \mathrm{~T}$ and thus helps bridge the low-field region between the positron accumulator and the main ATHENA magnet (see fig.1) while also making it possible to separate the two vacuum systems with a pumping restriction. The positrons are released by lowering a gate electrode with a fall time of $1 \mu$ s and trapped by closing another gate electrode in the main magnet, $3.2 \mu$ s later. This traps the positrons initially in the entire length of the mixing trap and the adjacent positron trapping section. The positron plasma is then subsequently axially compressed into the central harmonic region of the mixing trap. This compression takes a few tens of seconds. The overall efficiency for transfer, recapture and compression is about $50 \%$. It should be noted that this efficiency was found to be mainly limited by the electronics, particularly the closing time of the trapping electrode in the main magnet. Efficiency gains are anticipated when faster electronic units are installed. The present settings allow the delivery of about 75 million positrons for recombination every 5 minutes. The lifetime of the compressed positrons in the mixing trap is quite long as no significant loss was observed during a hold period of 4000 seconds.

We conclude this section by remarking that the operation of the positron accumulator, including the rotating wall compression, was found to be stable and reproducible over a wide range of operating parameters. Thus the size, density and position of the positron plasma can be predicted prior to transferring it to the main recombination trap.

\subsection{The CsI monitor}

A calibrated CsI crystal with photodiode readout measures the annihilation intensity when the positron beam is dumped on the segmented Faraday cup. The calibration was achieved using the $\gamma$-lines at $511 \mathrm{keV}$ and $1.274 \mathrm{MeV}$ from a weak ${ }^{22} \mathrm{Na}$ source of known activity. Since the $511 \mathrm{keV}$ line from positron annihilation is used to determine the number of positrons, contributions at low energies from the 1.274 $\mathrm{MeV}$ line (e.g. Compton scattering) need to be subtracted. This was achieved using a calibrated ${ }^{60} \mathrm{Co}$ source which emits $\gamma$ 's at 1.17 and $1.33 \mathrm{MeV}$, and hence produces a spectrum in the $511 \mathrm{keV}$ region similar to the ${ }^{22} \mathrm{Na} 1.274 \mathrm{MeV}$ line.

The sources were placed in turn inside the positron accumulator at the position of the segmented Faraday cup so that conditions were as close as possible to those experienced during the experiment. In particular the $\gamma$ 's produced a spectrum sim- 
ilar to that of the Compton scattered photons from the annihilation of the positron plasma.

Two quantities were extracted from the background-corrected spectra of the 511 $\mathrm{keV}$ line: (i) the average energy per photon and (ii) the overall efficiency of the detector. The average energy per photon is used to calculate the number of positrons annihilating on the Faraday cup during a standard plasma dump. In its operating position, the CsI photodiode detector had an efficiency of $(0.049 \pm 0.006) \%$ including solid angle acceptance.

Two further CsI photodiode detectors were used to monitor the transfer of the positron plasma from the positron accumulator to the mixing trap. One was situated so it particularly observed any losses in the transfer section and the other was situated directly outside the main magnet (see section 2.1) at the position of the degrader foil where the positron plasma was dumped. These detectors were then calibrated by a similar procedure to the main CsI diode detector by placing sources at suitable positions inside the apparatus and measuring the resulting detector response, thus again allowing for any material dependent attenuation. These detectors made it possible to optimize the positron transfer independent of the main ATHENA detector (see section 8 ) and provided an independent way of crosschecking the degrader Faraday cup calibration for positrons.

\section{The ATHENA Antihydrogen detector}

In a homogeneous magnetic field electrically neutral $\bar{H}$ atoms escape the confinement region and annihilate on the trap electrodes producing on average about three charged pions, three high energy $\gamma$ 's and two $511 \mathrm{keV} \gamma$ 's (fig. 21).

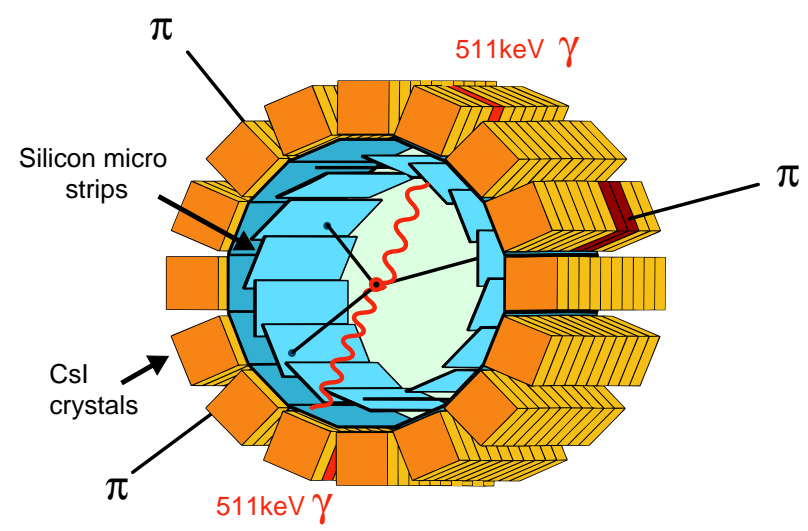

Fig. 21. Sketch of $\bar{H}$ annihilation on the trap wall (not shown). Solid lines represent charged pions, wavy lines photons from positron annihilation. The crystals hit by the charged pions or $511 \mathrm{keV}$ gamma photons are indicated. High energy $\gamma$ 's from $\pi^{0}$-decay are not shown. 
The detector [53,54] was designed to allow extraction of a clean $\bar{H}$ signal for background rates of up to $10 \mathrm{kHz}$, although the effective background rate was later found to lie below $100 \mathrm{~Hz}$. Charged particles are detected in two layers of $\mathrm{Si}-\mu$-strip detectors covering roughly $80 \%$ of $4 \pi$. A three dimensional reconstruction of the $\bar{p}$ annihilation vertex is achieved with $\sigma=4 \mathrm{~mm}$ spatial resolution by straight line extrapolations of the charged particle tracks. Photons from positron annihilation (511 keV) convert in the CsI crystals via the photoelectric effect with a probability of about $25 \%$. The reconstruction efficiency for antihydrogen events can, in principle, be increased if Compton scattering is also included. The segmentation into 192 crystals is required to ensure high enough angular resolution to verify that the two $511 \mathrm{keV}$ photons are emitted back-to-back. High granularity is also imposed by background considerations in $\overline{\mathrm{p}}$ annihilation. The annihilation releases $2 \mathrm{GeV}$ of center-of-mass energy which is distributed among the pions and later high energy $\gamma$ 's. With 192 crystals the probability of a crystal being hit by either a pion from the annihilation vertex or one of the high energy $\gamma$ 's is about 5\%/crystal. This background contains (spatially uncorrelated) $511 \mathrm{keV}$ photons produced by $\gamma$ showers in the surrounding apparatus (e.g. magnet coils). This background signal will always be present in our antihydrogen signal but it does not exhibit the angular correlation of $180^{\circ}$ that the two $511 \mathrm{keV}$ photons from the annihilation of antihydrogen produce.

\subsection{Design and realization of the detector}

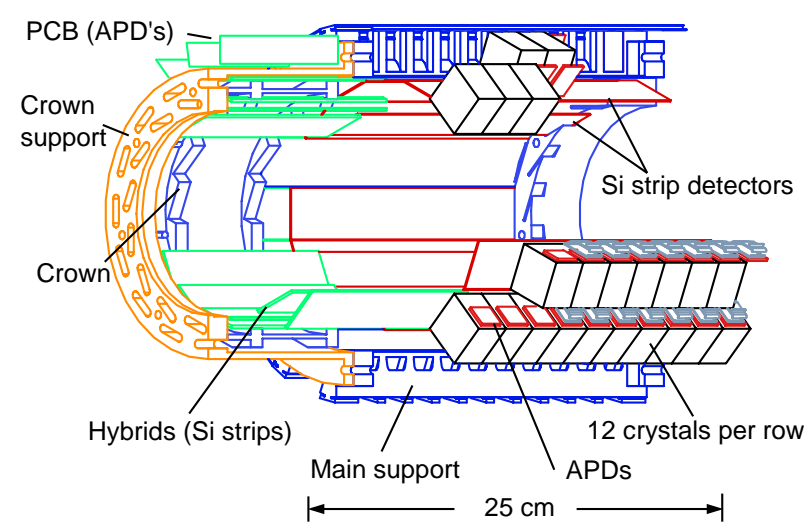

Fig. 22. 3-dimensional drawing of the $\bar{H}$ detector.

The most stringent constraint on the detector design is the low operating temperature of $140 \mathrm{~K}$, determined by its location a few millimeters from the cold $(15 \mathrm{~K})$ trap region in the center of the experiment. Low temperature is, in principle, advantageous for semiconductor detectors since parallel noise from leakage currents practically vanishes, but constraints increase on mechanics, cabling and electronics. It also prevents easy access for debugging in final working conditions. Figure 22 shows the cylindrically symmetric design of the detector operating in a vacuum of $10^{-7}$ mbar. The overall dimensions are 75 (140) $\mathrm{mm}$ inner (outer) diameter and 250 
$\mathrm{mm}$ in length. The outer diameter is limited by the cold bore of the superconducting solenoid. The inner diameter is limited by the size of the cryogenic coldnose UHV vessel containing the electrodes of the nested Penning trap (see section 2.1). The inner-most part of the detector of thickness less than $10 \mathrm{~mm}$ contains two layers each of 16 double sided silicon $\mu$-strip modules, $162 \mathrm{~mm}$ long. Separation between the modules and the 192 crystals is provided by the $500 \mu \mathrm{m}$ thin aluminum wall of the main support, which was manufactured from a single piece of aluminum using electro-erosion. The crystals with dimensions $17 \times 17.5 \times 13 \mathrm{~mm}^{3}$ are grouped in 16 rows of 12 , closely filling the remaining space.

The $\mu$-strip modules consist of two double sided sensors (SINTEF, Norway, $81.6 \times$ $19 \mathrm{~mm}^{2}$ with a wafer thickness of $380 \mu \mathrm{m}$ ) glued onto a silicon mechanical support, and a multilayer ceramic hybrid, $2 \mathrm{~mm}$ thick (fig. 23).

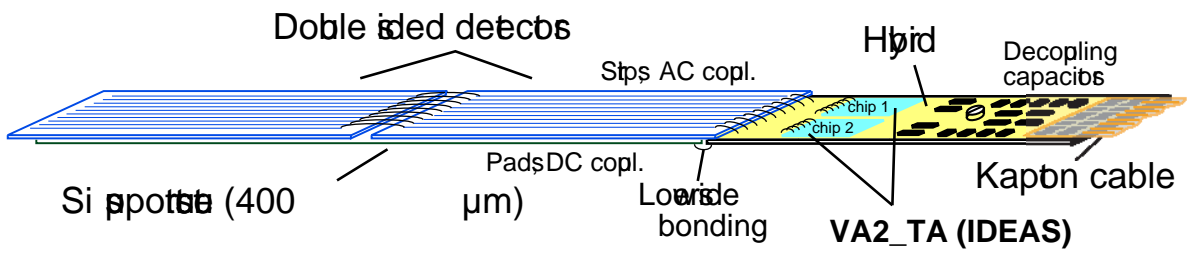

Fig. 23. Silicon $\mu$-strip module.

The sensor p-sides are segmented into 384 AC coupled strips with an implant width of $32 \mu \mathrm{m}$ and a pitch of $46.5 \mu \mathrm{m}$. Every third strip is read out while the two intermediate strips are floating. The readout strips $(139.5 \mu \mathrm{m}$ pitch) are bonded to the pitch adapter integrated in the hybrid, itself bonded to a 128 channel VLSI chip (VA2_TA) glued on the hybrid (see section 8.2$)$. The 64 DC coupled pads $(1.25 \times$ $18 \mathrm{~mm}^{2}$ ) of the sensor $\mathrm{n}$-sides are oriented perpendicular to the strips. Thin gold plated aluminum lines on the silicon support deliver the 128 signals to the bottom of the hybrid from where they are brought to a second chip VA2_TA via 128 feedthroughs in the ceramic. For simplicity, a common ground is used for both readout chips. This requires the depletion voltage $(-65 \mathrm{~V})$ to drop on the $\mathrm{AC}$ couplings of the sensor strips. A fraction of $3 \%$ of the strips had a short circuit to the implant and had to be disconnected. However, they could still deliver signals by capacitive coupling to adjacent strips.

Pure CsI scintillation crystals obtained from CRISMATEC (France) are used to detect the $511 \mathrm{keV}$ photons. The light yield is excellent at low temperature $(\approx 50,000$ photons $/ \mathrm{MeV}$ at $80 \mathrm{~K}[55])$, and the decay time $(\approx 1 \mu \mathrm{s})$ fits the shaping time of the front end electronics. The crystals were originally coupled to $17 \times 13 \mathrm{~mm}^{2}$ photodiodes covering the front of the crystals. For cost reasons they were produced on the same wafers as the silicon strip sensors, requiring only one additional mask for the shallow n-implant on the light sensitive side. However, only a modest signal-overnoise ratio $(\approx 15$ for $511 \mathrm{keV}$ absorption) could be achieved due to the unavoidable serial noise, electric contact problems, difficult optical coupling and low blue light sensitivity. These diodes were then replaced by avalanche photo diodes (APD, 


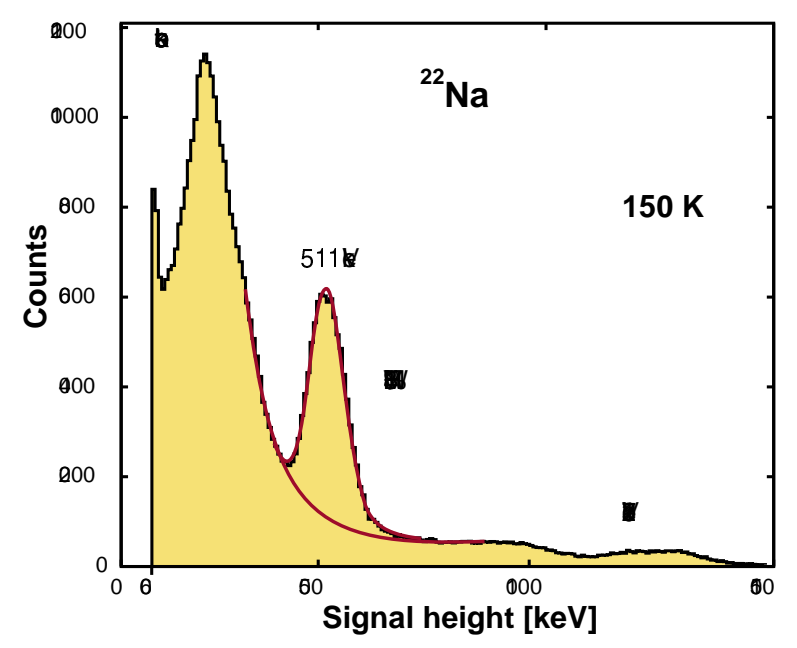

Fig. 24. ${ }^{22} \mathrm{Na}$ energy spectrum measured by one crystal equipped with an APD.

Hamamatsu, type $S 8148,5 \times 5 \mathrm{~mm}^{2}$ ), obtained from the electromagnetic calorimeter project of the CMS collaboration, after carefully testing their low temperature functionality. From 300 diodes not satisfying quality criteria for CMS, 220 were selected for operation at a gain of $20 \pm 3$, at $135 \mathrm{~K}$ and $230 \mathrm{~V}$ bias voltage. A typical signal-over-noise ratio of larger than 50 was achieved in spite of the smaller active area of the APDs, which is the limiting factor for the energy resolution $(\simeq 18 \%$ FWHM at $511 \mathrm{keV}$, fig. 24).

The APDs (capacity $C_{D} \approx 80 \mathrm{pF}$ ) or the old photodiodes are also read out with VA2_TA chips. One chip is located at the end of a $230 \mathrm{~mm}$ long and $12 \mathrm{~mm}$ wide printed circuit board (PCB) which connects the 12 APDs of one row to the 12 first input channels of the chip. A carbon fibre inlay was inserted into the PCB under the area supporting the chip to match the different thermal expansion coefficients.

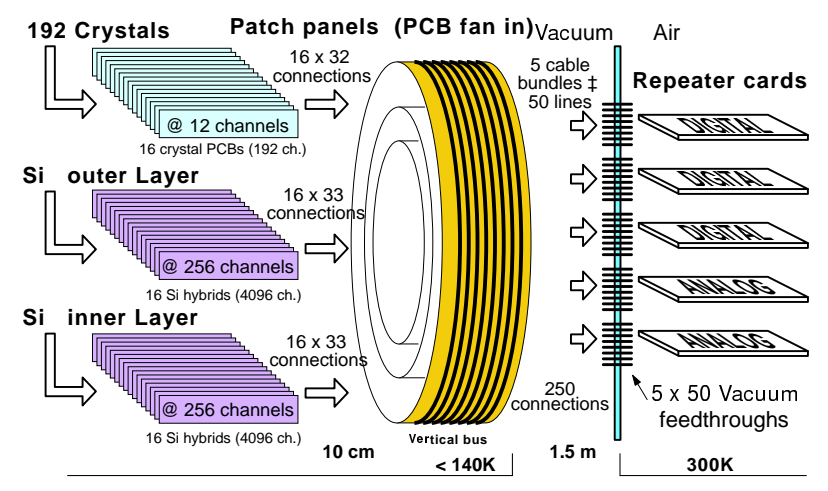

Fig. 25. Block diagram of the detector (see text).

The 16 PCBs of the crystals and the 16 ceramic hybrids of each silicon $\mu$-strip layer are connected in parallel by $8 \mathrm{~cm}$ long kapton cables (33 lines) each to a cylindrically bent $0.5 \mathrm{~mm}$ thick printed circuit board. This arrangement divides the detector into three independent subgroups, or patch panels (fig. 25). Each patch panel (at $140 \mathrm{~K}$ ) is connected by a $1.5 \mathrm{~m}$ long bundle of 50 shielded cables (LakeShore $1 \mathrm{~mm}$ 
diameter $50 \Omega$ cryo-cables with drain wire and aluminium foil shielding) to a CF150 vacuum flange at room temperature housing five 50-pin D-Sub feedthroughs.

Outside the vacuum vessel, five repeater cards are plugged directly into the flange, which also serves as the main grounding point. Three of these boards, the digital repeater cards, house the main electronics for controlling the three subdetectors. They contain a low noise power supply $( \pm 2 \mathrm{~V})$, some logic circuitry (GALs) and level translators, 16 voltage-DACs for chip settings, bipolar calibration pulse generators, the receiver for trigger signals (currents) and a programmable window discriminator (trigger multiplicity). The cards are connected by two digital busses to VME modules. A sequencer (Caen V551B) controls the readout and ADC sampling by 8 differential TTL lines, while a 16 bit ECL digital I/O unit (Caen V262) is used for programming trigger masks, serially loaded DAC registers and other logic states of the detector, such as calibration and test mode.

Since no other active electronics could be placed in the cold section of the detector, the differential current output buffers of the chips drive the multiplexed analog signals over $1.5 \mathrm{~m}$ long cables of the same type as the digital lines. These signals are fed into receivers on two analog repeater cards on the vacuum flange. One pair of lines is used for each of the 256 channel $\mu$-strip modules, while the 16 crystal PCBs share four lines. Differential line drivers send the 36 analog signals to 18 double channel FADCs (Caen V550) located in the same crate as the other VME modules. The crate is connected via an MXI-2 bus (National Instruments) to a PC in the ATHENA control room running LabView as DAQ software. Data (40 kByte/event) can be written to disk with a rate of typically $100 \mathrm{~Hz}$, mainly limited by the $5 \mathrm{MByte} / \mathrm{s}$ transfer rate of the VME - PC link.

Shaping times for both detector components are set to $3 \mu \mathrm{s}$, adapted to the time constant of light emission for pure-CsI [55]. The read out controller freezes all 8384 analog channels simultaneously and provides a fully synchronised readout, event by event. The total power consumption amounts to roughly $5 \mathrm{~W}$. The multiplicity coded trigger lines from the individual subdetectors (one line from each subdetector) can, however, be recorded independently, but have to be masked during the chip readout time $(150 \mu \mathrm{s})$, due to digital cross talk. The time jitter is typically 120 ns for the $\mu$-strips and $300 \mathrm{~ns}$ for the crystals.

\subsection{The read out chip VA2_TA}

The trigger on pure photon events from $\mathrm{e}^{+}$annihilations is an essential component of the detector. The trigger signal is also needed to monitor the high instantaneous annihilation rates of antiprotons and positrons and for general diagnosis without full read out of the analog signals.

The VA2_TA chip (fig. 26) is based on a series of CMOS VLSI chips (Viking [56], 


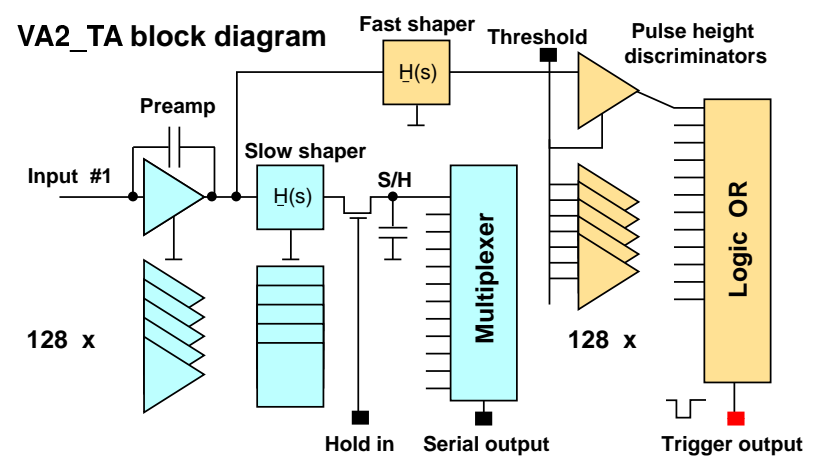

Fig. 26. Block diagram of the VA2_TA chip.

VA), already well established in high energy physics applications. A 32 channel version (VA32_TA32 set of separated analog and trigger chips) was first successfully tested at low temperatures.

A 128 channel chip with a medium scale input stage $\left(60+11 / \mathrm{pF} \mathrm{e}_{r m s}^{-}\right)$, a standard shaper $(\approx 2 \mu \mathrm{s})$ and a second faster shaper $(\approx 75 \mathrm{~ns})$, was developed for our application by Ideas ASA, Norway. The outputs of the fast shapers are fed to 128 discriminators (see fig. 26) with common threshold and programmable transition polarity. A register is loaded to disable noisy channels. Power consumption, shaping times, threshold voltages or output signal heights are controlled by 12 DC levels. These settings are generated as voltages by the DACs on the digital repeater cards and are common to all subdetectors. Figure 27 illustrates for instance the spread in shaping times for the crystal readout, as a function of temperature. Optimising settings lead to an average shaping time of $3 \pm 0.5 \mu$ s at $140 \mathrm{~K}$.

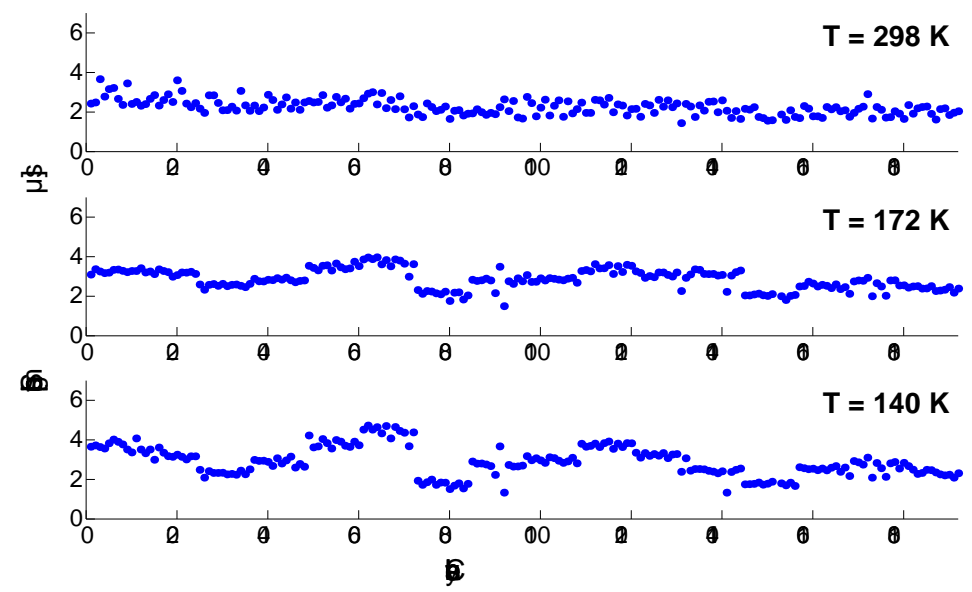

Fig. 27. Temperature dependence of the peaking time for the crystal shapers.

Some of the chip settings, in particular the input transistor current of the preamplifier, are quite sensitive to noise down to $\mu$ volts. Therefore ceramic decoupling capacitors and RC filters are used on all DC lines of the hybrids (PCBs). Some capacitors had to be oversized by up to $500 \%$ to take into account the strong ferroelectric behaviour of dielectrica under DC operation at low temperatures. Power 
for the digital part of the chip is taken locally from the analog supply via RC filters $(10 \Omega, 20 \mu \mathrm{F})$, while further $20 \mu \mathrm{F}$ tantalum capacitors buffer the analog power.

Global grounding was optimised empirically by removing or setting various ground bridges on key positions of the detector. The two grounds of the digital and analog parts in the chip were connected directly to the ground plane on the hybrids and PCBs. Stable working conditions were observed with no observable cross talk from firing triggers nor common mode noise.
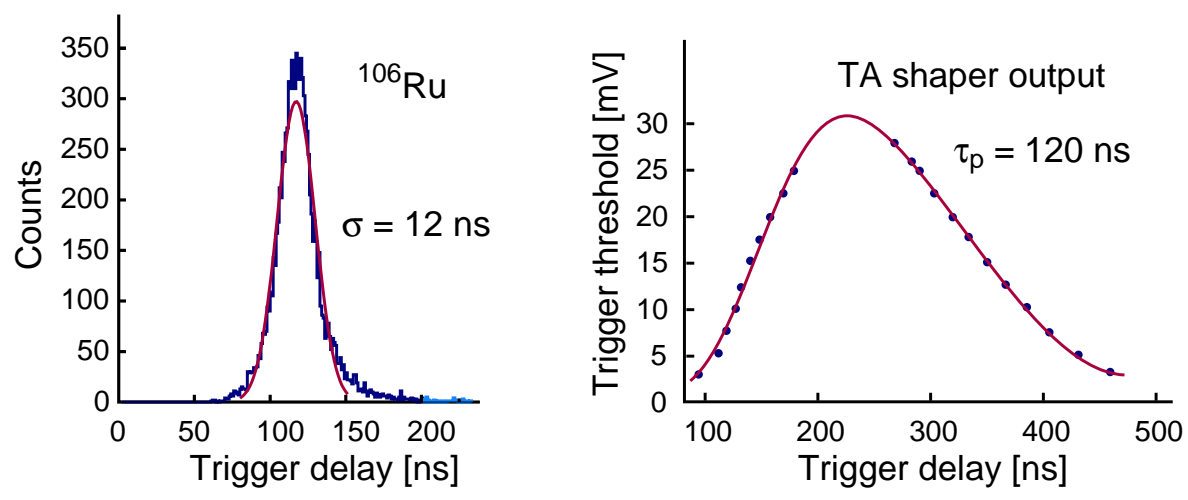

Fig. 28. Timing resolution (left) and shaper output (right) of the VA2_TA chip.

Due to ohmic resistances in the service cables (the 250 shieldings are connected to a single ground) and the fully floating operation with respect to the rest of the ATHENA apparatus, the detector ground may be as high as $50 \mathrm{mV}$. Sensitive voltages such as the trigger threshold are corrected for this offset by voltage dividers referencing to the hybrid (PCB) grounds. Various performance measurements were made on the VA2_TA chips. The trigger timing resolution at room temperature from a small test detector $\left(C_{D} \approx 20 \mathrm{pF}\right)$ with respect to a plastic scintillator coupled to a photomultiplier is shown in fig. 28 (left). Figure 28 (right) shows the output signal of the fast shaper (TA), recorded by scanning the trigger delay over trigger threshold. A peaking time $\left(\tau_{p}\right)$ of $120 \mathrm{~ns}$ was thereby determined for the fast shaper.

\subsection{Position resolution of the $\mu$-strip modules}

The signal-to-noise ratio of the $\mu$-strip modules is about 40 on the strip and 50 on the pad side, respectively, measured with minimum ionizing particles at low temperatures. The somewhat higher noise on the strips, in spite of the p-type implants, is caused by the large ratio of strip implant to pitch widths, leading to a rather large interstrip capacitance of $\approx 2 \mathrm{pF} / \mathrm{cm}$. However, a good efficiency for charge collection on the strips was measured with cosmic rays and a high precision $(\sigma \approx 1 \mu \mathrm{m})$ incident telescope [57]. Charge loss on the floating strips was negligible. Cosmic data could also be used to determine the spatial resolution on the strip side. Figure 29 shows the $\eta$ distribution, where $\eta$ is defined as the fractional charge collected on 
one of the two adjacent readout strips $\left(\eta=\frac{Q_{L}}{Q_{L}+Q_{R}}\right)$. The two central peaks are due to capacitive coupling of the intermediate floating strips to the readout strips. The differences between predicted hit positions from the incident telescope and measured position, using the weighted average of adjacent strips, are plotted in fig. 29. We obtained a position resolution of $\sigma=28 \mu \mathrm{m}$. However the much worse overall vertex resolution of approximately $4 \mathrm{~mm}(1 \sigma)$ in ATHENA is determined by the unknown particle track curvatures in the $3 \mathrm{~T}$ magnetic field of the trap region. This is because we extrapolate the tracks of the charged pions from only 2 points in the silicon $\mu$-strip detector and with just these two points and no information about the charge of the detected pion, we have no way of determining the track curvature.
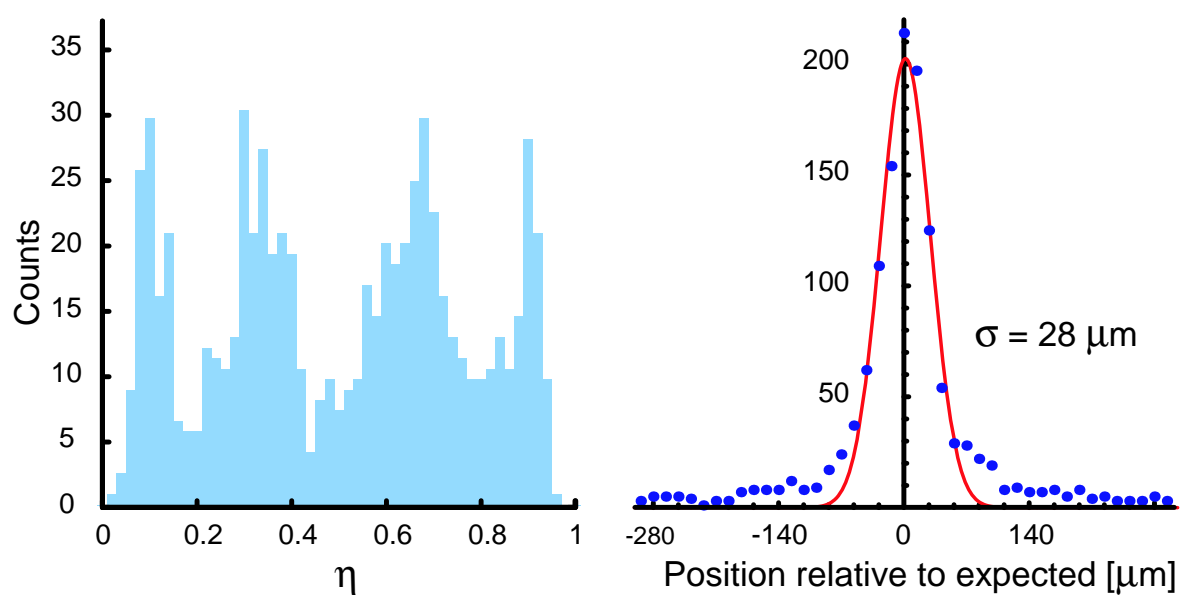

Fig. 29. $\eta$ distribution (left) and spatial resolution (right, see text).

\section{Control and data acquisition system}

The control system is required to control all 27 electrodes of the mixing trap and the 11 electrodes of the positron transfer section (see section 7.3), and to manage communication with the computers in charge of catching and cooling antiprotons and accumulating positrons when these particles are being transfered into the mixing trap.

Voltages on the electrodes of the mixing trap are supplied by programmable triggerable DAC's (three $100 \mathrm{kHz}$ 16-bit VME-based DACs (Joerger VDACM) with a buffer depth of 32k steps and one $1 \mathrm{MHz} 12$ bit PCI-based National Instruments DAC (6713) with a buffer depth of 16k steps). Voltages to the transfer electrodes, as well as triggers to the DACs, are supplied by two PCI-based $10 \mathrm{MHz}$ programmable and triggerable 32-channel pulse generators (Becker \& Hickl PPG-100) with a buffer depth of $64 \mathrm{kB}$. Additional fast (rise time $<100 \mathrm{~ns}$ ) pulses of arbitrary shapes are provided by four $100 \mathrm{MHz} 12$ bit VXI-based waveform generators (RACAL Instr. 3151 ), which can be connected to any of the 27 mixing trap electrodes. 
The interface to the control system is written in LabVIEW; in combination, the pulse generator and the DAC's are able to provide the mixing trap and transfer electrodes with voltage sequences consisting of several thousand steps and lasting several hours with microsecond precision. In addition, these sequences contain provisions for further activities such as removing electrons from the transfered antiprotons, controlling the status of the electron source, and generic triggers and gates. Once created, these sequences are downloaded to the DACs and pulse generators, leaving the time-critical execution under hardware control. The voltage range of the DACs and pulse generators is increased to $\pm 140 \mathrm{~V}$ by amplifying all voltages with fast $(\sim 1 \mu \mathrm{s})$ pre-amplifiers. All voltages with the exception of those provided by the RACAL DAC's are filtered by a $10 \mathrm{MHz}$ low-pass filter to reduce noise; each electrode has an additional capacitively coupled unfiltered input for plasma diagnostics. This input is used on two electrodes to apply $40 \mathrm{~V} / 100 \mathrm{~ns}$ pulses generated by a $30 \mathrm{MHz}$ GPIB-based pulser (SRS DS345) to mixed antiproton-electron clouds, in order to efficiently clean the antiprotons of electrons (at least $95 \%$ of the electrons are eliminated with 4 pulses). Transfers of antiprotons and positrons are managed by setting up dedicated sequences of voltages which are triggered by the remote computers, once handshaking has been established, at which point sequences steering the mixing of antiproton and positron clouds take over. These procedures are fully automated, and can repeat the same experiment an arbitrary number of times.

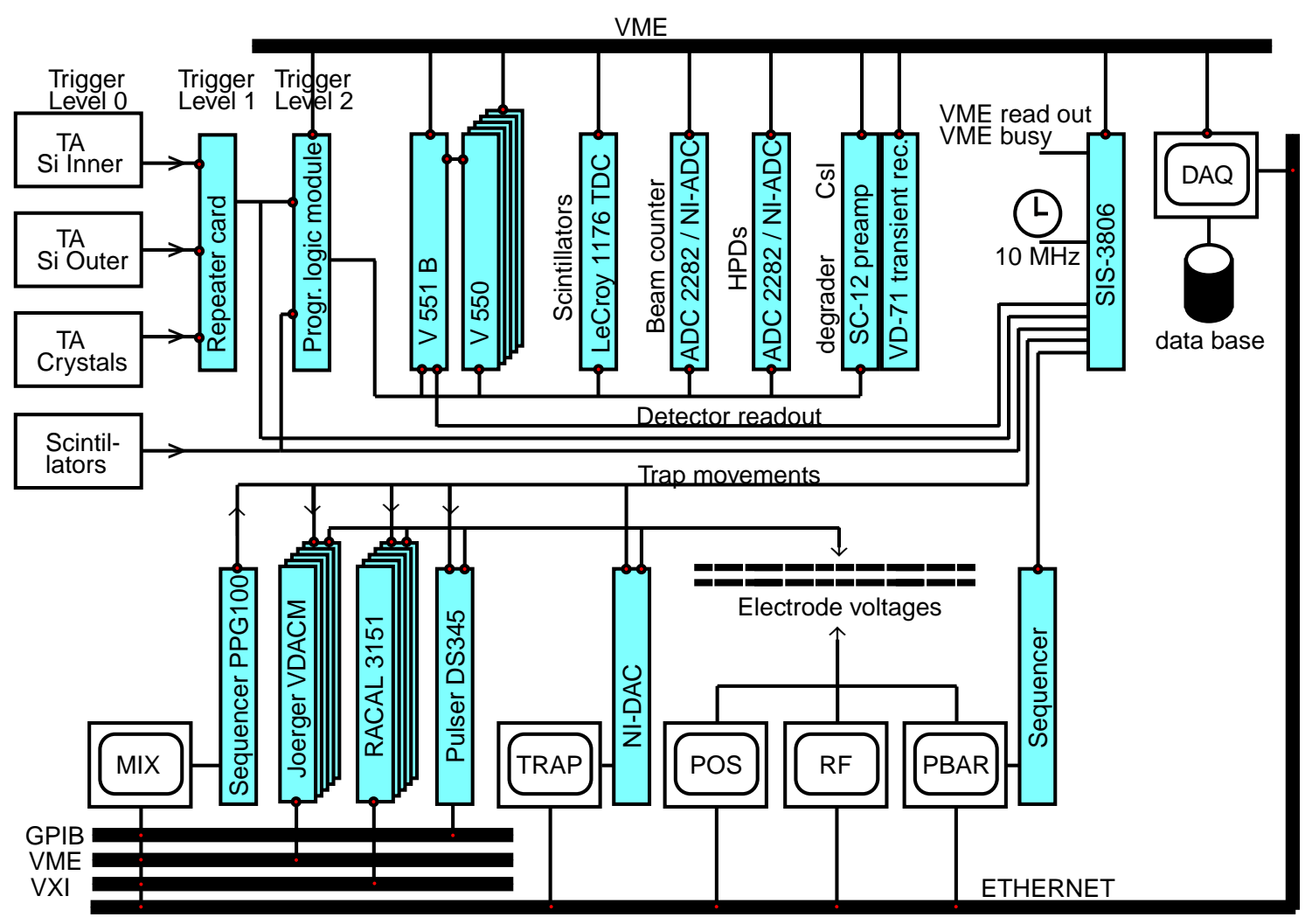

Fig. 30. Diagram of the data acquisition system.

The data acquisition system (DAQ, fig. 30) produces a record of all activities in 
the apparatus: standard detector read-out, changes in the voltages of the electrodes, state of the apparatus, voltage and timing information from the control system, and inter-computer communications. In addition to the central detector readout (see section 8), scintillator pulse heights (determined by LeCroy 1182 ADCs), pulse shapes (recorded by INCAA VD71 transient recorders), and oscilloscope signals are acquired via VME and GPIB modules readout by a LabView program, and written to a data base. The order in which the data are written to the data base does not necessarily correspond to the order in which events occur, since several modules contain multi-event buffers, and the control sequence for voltages and timings (which is downloaded to the data base before being executed) is not measured in real time. To retroactively establish timing, the heart of the DAQ consists of multi-hit time stamp units (one 8-channel and one 32-channel Struck SIS 3806) which record with up to $1 \mu$ s accuracy the time of each type of activity. Dead time-free acquisition is guaranteed internally by the use of two counter banks, one of which is active at any given time, while the data of the inactive bank is piped into a 64k FIFO. Switching between the two banks, which count the number of $100 \mathrm{~ns}$ intervals between switches, is triggered by an OR of all triggers. Triggers are generated by a 16-input VME general purpose programmable logic module (GPPLM, trigger level 2) combining all detector signals; this module allows the simultaneous construction of a variety of trigger conditions.

This information is then used by the offline data analysis program to establish trigger rates, correlations of one type of event (e.g. antiproton annihilation rate) with another type (e.g. voltage changes on the electrodes), time ordering of activities and detector dead times. Although the VME read-out rate itself is bandwidth limited (corresponding to a detector read out rate of about $100 \mathrm{~Hz}$ ), trigger rates up to a few $\mathrm{MHz}$ are reliably recorded for 32 types of triggers in parallel, and with a time resolution of $10 \mathrm{~ms}$, by the 32-channel time-stamp unit. A higher time resolution of $1 \mu \mathrm{s}$ is achieved for a further eight special interest triggers with the 8-channel time-stamp unit.

\section{The online software}

The ATHENA online system was developed in $\mathrm{C}++$ by using the ROOT package [58]. Data from the apparatus are analyzed in real time and saved on media by the DAQ program. As described previously, data acquisition is performed in LabVIEW on a Windows NT machine and the data are streamed to disk via a Dynamic Link Library written in $\mathrm{C}$. The data are saved in a raw file by using a private binary format without any compression to obtain the maximum possible speed during acquisition.

The online system consists of two $\mathrm{C}++$ programs: (i) a monitoring program (AtOM, ATHENA On-Line Monitor), that reads the raw data from the DAQ and processes 
them, and (ii) an inspection utility (AtVIEW) for a quick analysis of the raw data during the runs. Because data are saved asynchronously as they are recorded by the detectors, the online software uses a hardware time stamp to re-order the events in the correct time sequence, to correlate data from the traps with data from the detectors.

The program also fills a set of preselected histograms used to monitor the experiment and saves the data as a ROOT tree in a TOF file (TOF, Time Ordered File). This ROOT file is compressed by a factor of about three with respect to the raw file. The results of the online analysis are also posted on the web for remote monitoring and are stored on tape at CERN via the CASTOR (Cern Advanced STORage) server.

The ROOT data tree is then passed to the offline software that extracts the detector events from the tree and performs the vertex reconstruction and the event analysis, as will be explained in section 11 .

Hence for each run three files are generated: (i) the raw data produced by the DAQ, (ii) the TOF file produced by the on-line, and (iii) the file produced by the offline programs

\section{The offline reconstruction software}

The ATHENA Off-line software is written in C++ and uses a set of ROOT macros. It decodes the detector response, reconstructs the interaction points of the particles in the inner and external silicon layers, associates the tracks to these points (pattern recognition), finds the vertex of the charged particles and selects the crystals with $511 \mathrm{keV} \gamma$-signals. A ROOT online monitor provides a display of the reconstructed events. Some batch macros give a set of histograms to control both vertex positions of the annihilations and detector performances.

A Monte Carlo (MC) code has been written to study the optimum selection criteria for $\bar{H}$ events and to evaluate the probability that a selected $\bar{H}$ event may be a background event. The chain for processing MC events is identical to that for real data.

The MC program was conceived as a $\mathrm{C}++$ interface between ROOT and the GEANT code for the generation of simulated data from nuclear physics experiments [59]. This interface allows the call of the FORTRAN routines of GEANT using ROOT macros. The ATHENA apparatus geometry was implemented in the MC code using the standard GEANT routines and copied into ROOT using a set of specialized routines. A phase space event generator was written, with the possibility to generate $p \bar{p}$ and/or $e^{+} e^{-}$annihilation events, in an uncorrelated or space-time correlated way. 
The $p \bar{p} \rightarrow$ mesons annihilation reactions considered, together with their branching ratio, are taken from ref. [60]. It is possible to call for one channel in particular (for example $p \bar{p} \rightarrow \pi^{+} \pi^{-} \pi^{+} \pi^{-}$) or to generate an annihilation event according to its branching ratio.

It should be noted that in reality $\bar{p}$ annihilations occur on nuclei of the trap electrodes or of the residual gas filling the trap volume, rather than on free protons. Although neutral pion multiplicities on complex nuclei are uncertain, a reduction of $20-40 \%$ with respect to the $\bar{p} p$ case is expected [61]. Therefore, the background effects due to the electromagnetic showers of high energy $\gamma$ 's in the magnet surrounding the traps and the detector are probably slightly overestimated in our MC.

Finally, the efficiency of each one of the 32 silicon modules and the energy response of each one of the 192 crystals have been implemented into the MC code, to fully simulate the performance of the real detector.

\subsection{Point reconstruction}

The hit coordinates of charged particles from the silicon $\mu$-strip modules are found by standard clusterization algorithms applied to the pedestal subtracted ADC values. The $(x, y, z)$ hit coordinates are calculated from the $(r-\phi)$-strip and $z$-pad information. Ghost points occurring when two or more particles hit the same microstrip module are eliminated in two ways: a) by keeping the hit configuration that minimizes the vertex residuals in three dimensions and $b$ ) by associating strips and pads that are correlated in amplitude when a module is hit by a single particle. Fig. 31 shows the correlation between strip and pad ADC contents. When a module has multiple hits, we consider those points as ghosts that have an ADC cluster contents out of the band indicated in the figure. It has been our experience that the best efficiency is obtained by using method a) when there are two hits on the module and method $b$ ) in the cases with more than two hits. The fraction of modules traversed by two or more particles is $13 \%$ and the ambiguity is resolved in $40 \%$ of the cases.

Fig. 32 shows the ADC amplitude distribution normalized to the traversed silicon thickness with cosmics (a) and for a standard run with antiprotons annihilating on the walls of the recombination trap (b). For cosmics good agreement is obtained with predictions assuming a Landau distribution, whereas the Landau fit to the annihilation data deviates at higher $\mathrm{dE} / \mathrm{dx}$ because of the presence of non-relativistic particles. Therefore, in fig. 32 (b) the Landau fit was restricted to the peak only (full curve). We recall that annihilation products are mostly pions of $300 \mathrm{MeV} / \mathrm{c}$ average momentum [60], with some kaons and nuclear fragments. It is these heavier nuclear fragments that account for the descrepancy between the Landau curve and the annihilation data for higher values of $\mathrm{dE} / \mathrm{dx}$. 


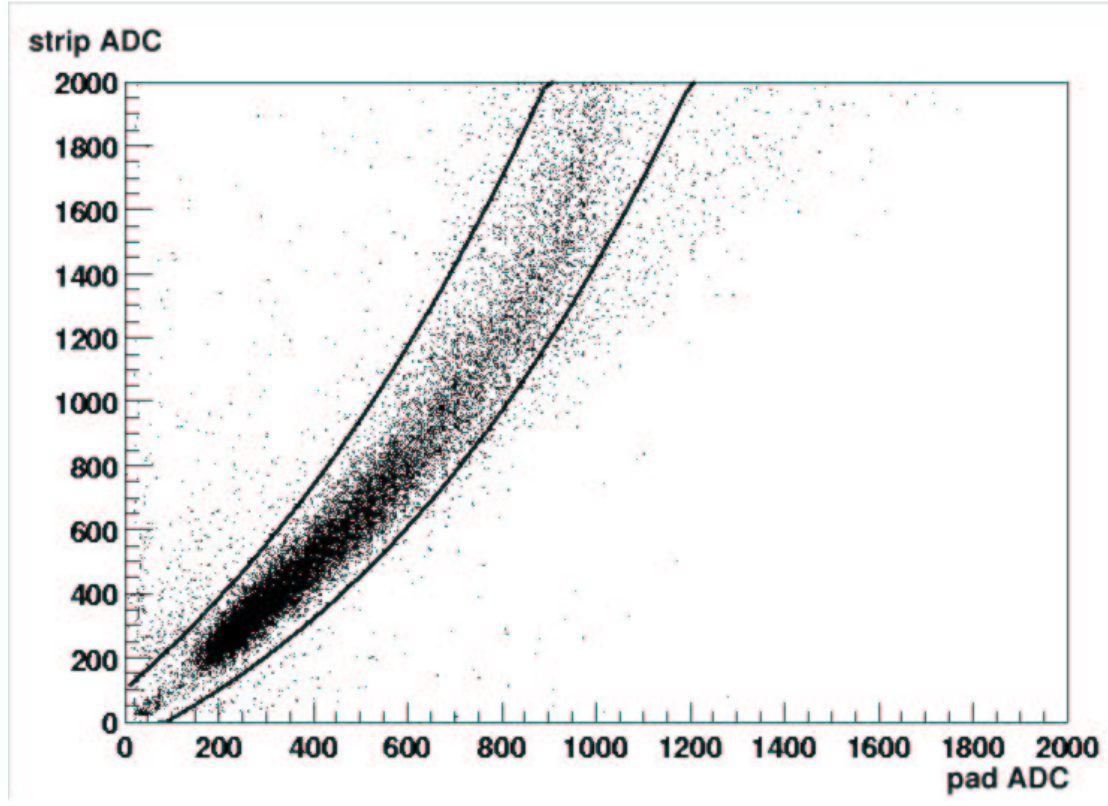

Fig. 31. Strip-pad correlation for silicon modules with one hit only. The two lines denote the boundaries of the correlation band used for method b) described in the text.
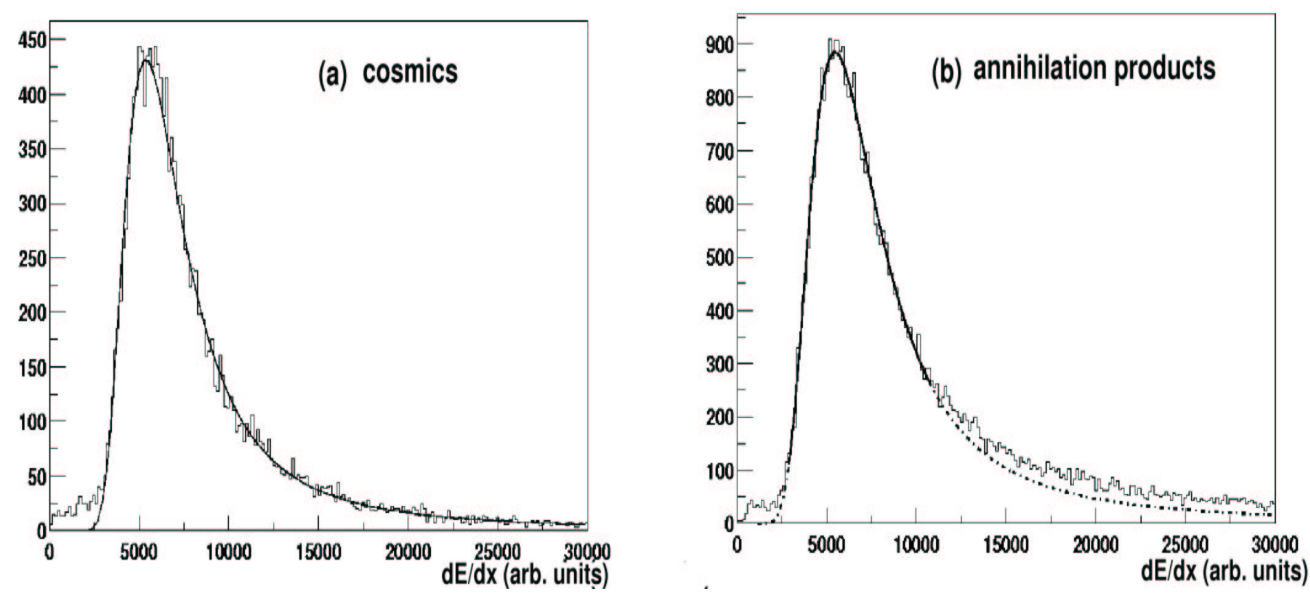

Fig. 32. ADC amplitudes per unit path length in silicon for cosmics (a) and for particles from $\bar{p}$ annihilations (b). The measurements were done using the strip side of the detector and for the cosmic data there was no magnetic field. The full curves show the fitted Landau distribution. The dashed curve in b) shows the prediction of the fit limited to the peak.

Assuming that the cosmic rays are $4 \mathrm{GeV}$ muons, the Landau most probable ionization loss is $\simeq 3.35 \mathrm{MeV} / \mathrm{cm}$. From the most probable value from fig. 32 (a) (5641 $\mathrm{ADC}$ counts $/ \mathrm{cm}$ ) one obtains a conversion factor of $\simeq 0.6 \mathrm{keV} / \mathrm{ADC}$ count.

The reconstruction software was tested by measuring the pitch between readout strips. The four cosmic ray hits obtained with no magnetic field were fitted to straight lines. The minimization of residuals as a function of a variable pitch in $r-\phi$ gave a readout pitch of $133 \mu \mathrm{m}$ and a mean residual of $240 \mu \mathrm{m}$, in very good 
agreement with the true pitch of $139 \mu \mathrm{m}$.

To detect antihydrogen a good performance of the crystal detector is crucial in order to assure correct reconstruction of the positron annihilation. During the experiments, the detector temperature was monitored continuously and the crystals were calibrated periodically with positron annihilation data coming from dedicated runs where the recombination trap was filled with positrons only.

The analysis of the 192 spectra from the individual crystals was performed in a semi-automatic way, with computer fits followed by the visual inspection of the displayed spectra, to maintain all the windows correctly around the $511 \mathrm{keV}$ signal. The optimal lower and upper window limits were found to be $345 \pm 20$ and $675 \pm 20$ $\mathrm{keV}$, where the uncertainties refer to the differences between the crystals.
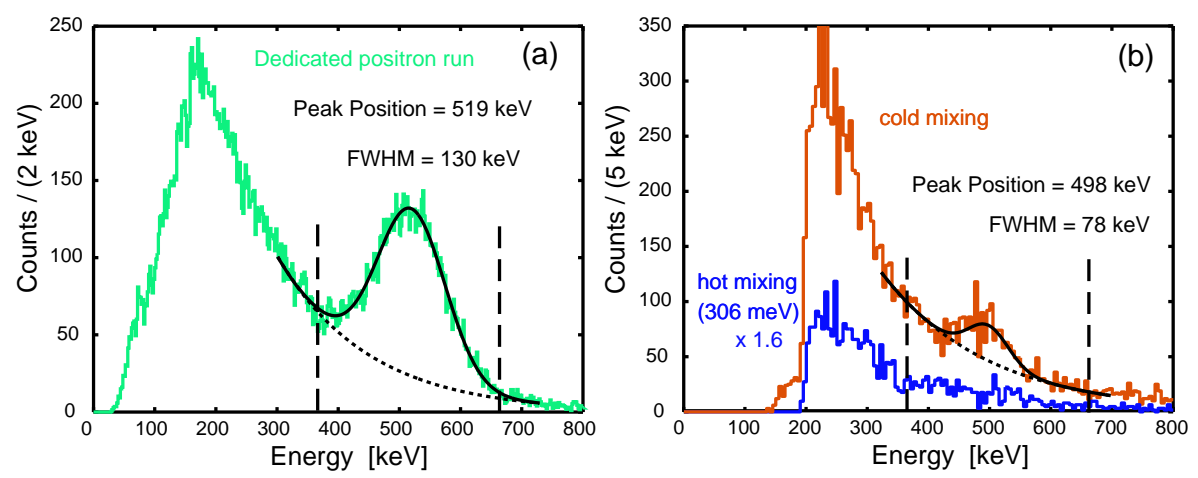

Fig. 33. A typical crystal spectrum in ADC count obtained during a dedicated positron run a) and during a normal run b). The lines indicate the limits of the $511 \mathrm{keV}$ window.

In fig. 33 typical crystal spectra obtained during the dedicated positron calibration runs and during normal data taking runs [1] are shown. The $511 \mathrm{keV}$ photon signals from the normal runs appear at the lower end of the energy spectrum, close to the noise (see also fig. 24), while minimum ionizing charged particles which deposit about $6 \mathrm{MeV} / \mathrm{cm}$ in the crystals and pair production from high energy $\gamma$ 's generate a flat plateau centered around $10 \mathrm{MeV}$. The background due to these $\bar{p}$ annihilation products is clearly visible in fig. 33(b). Therefore, the identification of the 511 $\mathrm{keV}$ signal from antihydrogen required the dedicated event selection procedure described in section 11.3. Concerning the stability of the whole detector, peak shifts of less than $5 \%$ over a 5 months period have been found for each of the 192 crystals.

\subsection{Event Reconstruction}

A very simple pattern recognition algorithm associates each point in the $r-\phi$ plane on the external silicon layer to the nearest point on the inner silicon layer. These two points define a charged particle track through the detector. Particular cases, like three-hit clusters, are considered as three-point tracks which pass through the overlapping inner modules (see fig. 35). 
A line passing through the two or three points is then calculated. A straight line fit is performed in the three point case, whereas in the two point case (about $95 \%$ of the tracks) the best fit equations are used only to propagate the errors from the hits on the track parameters, to estimate the accuracy of the vertex. As mentioned in section 8.3 track curvatures due to the magnetic field cannot of course be measured with two layers. Systematic effects deriving from the straight line approximation are discussed below.

We use two different vertex reconstruction algorithms, called the 3D (three - dimensional) and the dual algorithm, respectively. In the 3D algorithm the track is parameterized by the direction cosine vectors $\overrightarrow{\mathbf{c}}$ and the track length parameter $t_{i}$; the vertex coordinates are found from the $t_{i}$ values that minimize the sum of distances

$$
D_{\text {min }}^{2}=\min \left\{\sum_{i<j}\left|\mathbf{r}_{i}-\mathbf{r}_{j}+\mathbf{c}_{i} t_{i}-\mathbf{c}_{j} t_{j}\right|^{2}\right\} .
$$

After minimization, the vertex residual in three dimensions defined as

$$
R=\frac{D_{\min }^{2}}{\text { number of tracks to the vertex }}
$$

is an estimate of the resulting accuracy. Apart from this residual estimate, the 3D algorithm does not permit an easy error propagation, because of its intrinsic nonlinearity. However, knowledge of the vertex error is necessary for the definition of the vertex quality, an important parameter in event selection.

For this reason we also implemented the dual algorithm. When tracks have a common vertex $\left(x_{v}, y_{v}\right)$ in the $x-y$ plane, they obey the equation $y_{v}=a_{i} x_{v}+b_{i}$ which implies that $b_{i}=a_{i}\left(-x_{v}\right)+y_{v}$, is a straight line in the dual $a-b$ plane with coefficients $\left(-x_{v}\right)$ and $y_{v}$. This is true, with obvious change of notation, for each projection $x_{1}-y_{1}, y_{2}-z_{1}$ and $x_{2}-z_{2}$. For example, for the $x-y$ projection the vertex coordinates are obtained by minimizing the $\chi^{2}[62]$ :

$$
\chi\left(x_{v}, y_{v}\right)^{2}=\sum_{i=1}^{N} \frac{\left[b_{i}-\left(-x_{v}\right) a_{i}-y_{v}\right]^{2}}{\sigma^{2}\left(b_{i}\right)+x_{v}^{2} \sigma^{2}\left(a_{i}\right)-2\left(-x_{v}\right) \operatorname{cov}\left(a_{i}, b_{i}\right)}
$$

where the errors on $a_{i}, b_{i}$ stem from the propagation of the errors on the coordinates $x, y, z$ of the silicon strips and pads. In the absence of magnetic field these errors are given approximately by $\sigma_{x}=\sigma_{y}=\Delta_{\phi} / \sqrt{12}, \sigma_{z}=\Delta_{z} / \sqrt{12}$ where $\Delta_{z} \simeq 1.2$ $\mathrm{mm}$ and $\Delta_{\phi} \simeq 260 \mu \mathrm{m}$ are averages between the pitch $(\simeq 139 \mu \mathrm{m})$ and the silicon thickness $(\simeq 380 \mu \mathrm{m})$. No attempt was made to find the $x-y$ error dependence on the azimuthal angle, since the magnetic field effect is overwhelming. Indeed, 
realistic input errors are mostly due to the straight line fits that ignore the track curvatures in the magnetic field. By studying this effect with simulated data, we found that the effective $x-y$ pitch value, with magnetic field, is $\simeq 1.8 \mathrm{~mm}$. Therefore, the input errors have been fixed to:

$$
\begin{gathered}
\sigma_{x}=\sigma_{y}=7 \cdot \frac{\Delta_{\phi}}{\sqrt{12}}, \quad \Delta_{\phi}=260 \mu \mathrm{m} \\
\sigma_{z}=\frac{\Delta_{z}}{\sqrt{12}}, \quad \Delta_{z}=1.2 \mathrm{~mm} .
\end{gathered}
$$

After the fits in the three projections, each vertex coordinate gets two values with corresponding errors. The final coordinates of the vertex are given by their weighted averages. The comparison between the vertex coordinates $x_{1}, x_{2}, y_{1}, y_{2}$ and $z_{1}, z_{2}$ is a powerful tool to judge the quality of the vertex reconstruction.

We assign to each vertex a "Quality Index" (Q.I.) calculated by assigning a score to each of the following good vertex properties: 1) a small vertex residual $R$ (eqn. 2) in three dimensions: $R \leq 0.4 \mathrm{~cm}$; 2) a small discrepancy between dual and 3D algorithms: the vertices should agree within $4 \mathrm{~mm} ; 3$ ) a good matching of the $x_{1}-y_{1}, x_{2}-z_{1}, y_{2}-z_{2}$ projections in the dual algorithm; 4) a small vertex error in the dual algorithm $: \max \{\sigma(x), \sigma(y), \sigma(z)\} \leq 0.5 \mathrm{~cm}: 5)$ more than two tracks; 6) a small distance $(<0.1 \mathrm{~cm})$ between the inner silicon layer point and the line joining the external silicon layer point and the vertex.

The efficiency and accuracy of the vertex reconstruction have been studied with real and simulated data as a function of the Q.I. In table 2 the reconstruction efficiency of real and MC data is reported, together with the MC vertex resolution in space $(x-y-z)$ or in the $(x-y)$ projection only. The results for the $(y-z)$ and $(x-z)$ projections are similar. The resolution in the vertex reconstruction has been verified with real data through a sample of point-like $\bar{p}$ annihilations on the trap electrodes. The results, shown in fig. 34, are in very good agreement with the MC results of table 2. For the details of $\bar{p}$ annihilation imaging, see ref. [63]. A reconstructed real four prong annihilation event on the trap wall is shown in fig. 35 .

\subsection{Antihydrogen Event Selection}

The hit selection in the silicon $\mu$-strip detector and the vertex reconstruction strongly enhances $\bar{p}$ annihilation events with practically no background contamination. For the crystals, however, two main sources of background contribute: (i) short tracks from charged particles in the crystals and the conversion of high energy $\gamma$ 's lead to low energy background signals in the $511 \mathrm{keV}$ region; (ii) positrons from showers generated in the surrounding magnet by high energy $\gamma$ 's also lead to $511 \mathrm{keV}$ 

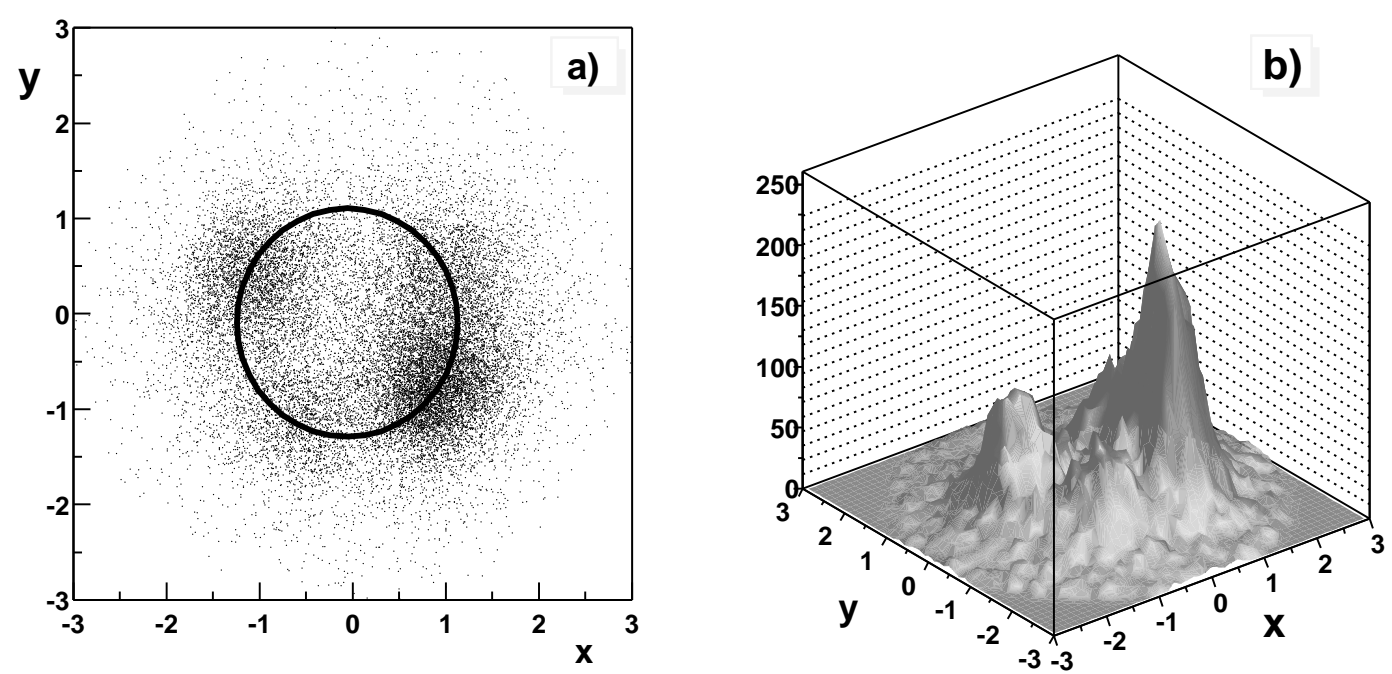

Fig. 34. Vertices reconstructed in the $x-y$ plane from point-like $\bar{p}$ annihilations on trap electrodes. The dimensions are in $\mathrm{cm}$. a):2-D view; the ring indicates the trap section (radius $=1.25 \mathrm{~cm}$ ). The measured width of the sharpest peak is $\sigma=0.5 \mathrm{~cm}$.; b): 3-D view of the histogram shown in (a).

Table 2

\begin{tabular}{ccccccc}
\hline Q.I. & Projection & $\eta(\mathrm{MC})$ & $\eta(\mathrm{real})$ & $\sigma_{x}(\mathrm{~cm})$ & $\sigma_{y}(\mathrm{~cm})$ & $D(\mathrm{~cm})$ \\
\hline 0 & $\mathrm{x}-\mathrm{y}-\mathrm{z}$ & $68 \%$ & $63 \%$ & 0.53 & 0.51 & 0.55 \\
0 & $\mathrm{x}-\mathrm{y}$ & & & 0.68 & 0.68 & 0.72 \\
\hline 6 & $\mathrm{x}-\mathrm{y}-\mathrm{z}$ & $13 \%$ & $7 \%$ & 0.30 & 0.32 & 0.38 \\
6 & $\mathrm{x}-\mathrm{y}$ & & & 0.37 & 0.39 & 0.47 \\
\hline
\end{tabular}

Quality of the reconstruction software with simulated annihilations on the trap walls (MC) and with real data, in space $(x-y-z)$ or in the $(x-y)$ projection only. $\eta$ is the vertex reconstruction efficiency, $\sigma_{x}$ and $\sigma_{y}$ are the r.m.s deviations of the distribution in $x$ ( or $y$ ) [reconstructed] - $x$ ( or $y$ ) [MC], and $D$ is the distance between generated and reconstructed $\mathrm{MC}$ vertices.

signals (see fig. 36). Our MC describes this background well, as shown in fig. 37.

These background effects have been greatly reduced by means of particular $\bar{H}$ selection criteria, studied by extensive Monte Carlo data. The procedure for antihydrogen event selection, which fully exploits the high granularity of the detector, is as follows. Only events with a reconstructed vertex are considered. Reconstructed tracks are extrapolated toward the crystals and those hit by an extrapolation line are identified and excluded from the subsequent $\gamma$ detection together with their 8 neighbors. Due to the limited efficiency, some tracks are not reconstructed and some isolated hits remain. Hence, also the crystals that lie above a hit on an external silicon layer module are considered as traversed by a charged particle and excluded as is its 8 neighbors. After this first selection, crystals are scanned for having a sig- 


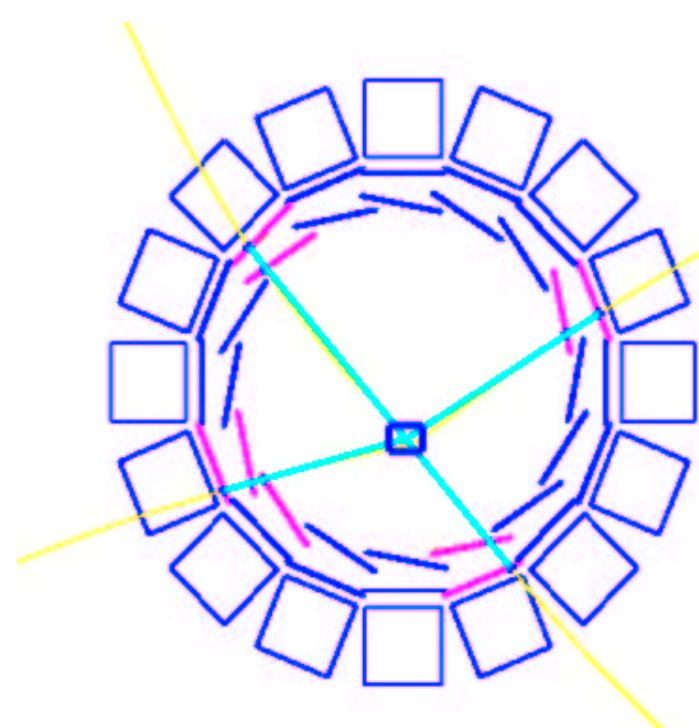

Fig. 35. Reconstructed antiproton annihilation event.

nal within the 350 - $550 \mathrm{keV}$ window and events with exactly two such crystals are selected. To reduce from this sample the fake $\bar{H}$ events due to the e.m. cascades from the surrounding magnet, also the requirement that the two selected crystals are not surrounded by other crystals with a signal above threshold is made. Therefore, the final sample consists of events with a reconstructed $\bar{p}$ annihilation vertex and two isolated crystals in the right energy window (called the candidate crystals), with the possibility to have some other crystals in the detector with signals outside this window.

The distance between the line joining the center of the two candidate crystals and the reconstructed vertex is expected to be zero in $\bar{H}$ annihilations. This corresponds also to the condition $\cos \theta=-1$ where $\theta$ is the opening angle between the two lines joining the candidate crystals and the $\bar{p}$ vertex (see also fig. 21).

Simulations of antihydrogen annihilations are based on the $\bar{p}$ and $e^{+}$annihilations occuring at the same time and at the same position on the wall of the recombination trap. We also considered two types of background, (1) due to a $\bar{p}$ that annihilates on a residual gas atom in the trap volume and (2) due to $\bar{p}$ and $e^{+} e^{-}$annihilations at the same time but in two different locations on the trap wall, simulating the - in terms of detection - worst case of an $\bar{H}$ atom that loses its positron before annihilating.

The MC efficiencies for the $\bar{H}$ annihilations and for these two types of background are shown in table 3 . The simulation shows also that the relative efficiencies between the $\bar{H}$ signal and the backgrounds are independent of the Quality Index of the reconstructed vertex.

The events and the signal in the table are defined as the number of entries in the opening angle histogram of figs. 38 and that in the first two channels, corresponding 


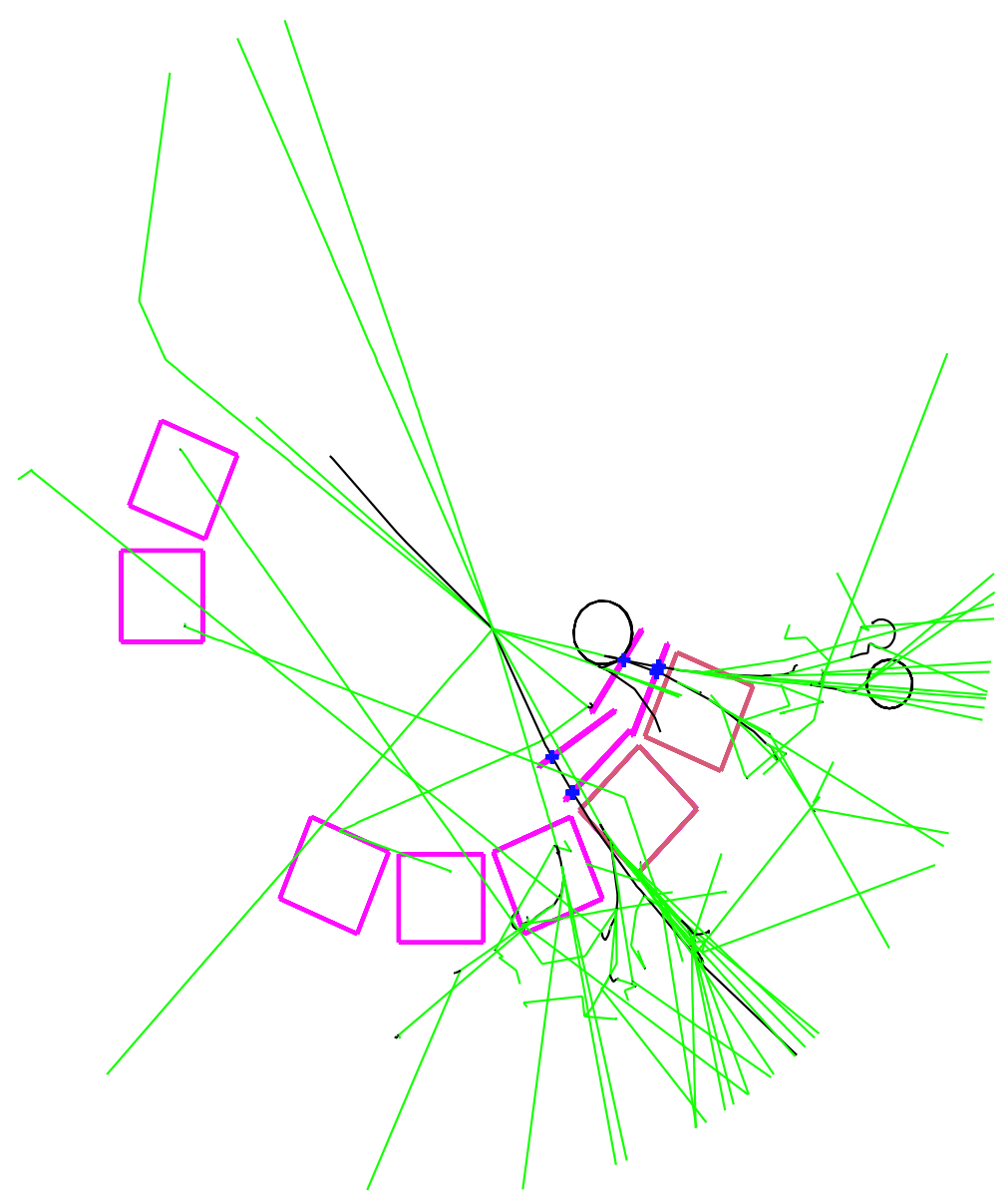

Fig. 36. A Monte Carlo event showing the noise generated on the detector by the high energy $\gamma$ showers in the surrounding magnet. Only the crystals with a $\gamma$ signal are shown.

\begin{tabular}{ccccc}
\hline \hline & & $\bar{H}$ events & $\bar{p}$ & $\begin{array}{c}(\bar{p})\left(e^{+} e^{-}\right) \\
\text {background (2) }\end{array}$ \\
\hline \multirow{2}{*}{ Entries } & MC & $(9.45 \pm 0.08) 10^{-2}$ & $(6.35 \pm 0.08) 10^{-2}$ & $(9.76 \pm 0.10) 10^{-2}$ \\
& data & & $(6.12 \pm 0.11) 10^{-2}$ & \\
\hline \multirow{2}{*}{ Signal } & MC & $(5.9 \pm 0.2) 10^{-3}$ & $(1.9 \pm 0.1) 10^{-3}$ & $(4.6 \pm 0.2) 10^{-3}$ \\
& data & & $(1.8 \pm 0.2) 10^{-3}$ & \\
\hline
\end{tabular}

Table 3

Real and Monte Carlo efficiencies for events entering into the opening angle histogram of fig. 38 (left) and into the first two channels around $\cos \theta \simeq-1$ following the selection procedure described in the text. The MC results are obtained starting from pure $\bar{H}$ annihilations on the trap wall, $\bar{p}$ annihilations in the open volume of the trap (background 1) and spatially uncorrelated $(\bar{p})\left(e^{+} e^{-}\right)$annihilations on the trap wall (background 2). The real data come from pure $\bar{p}$ annihilations on the trap wall.

to $-1 \leq \cos \theta \leq-0.947$.

The Monte Carlo histogram for the cosine of the opening angle is shown in fig. 38 

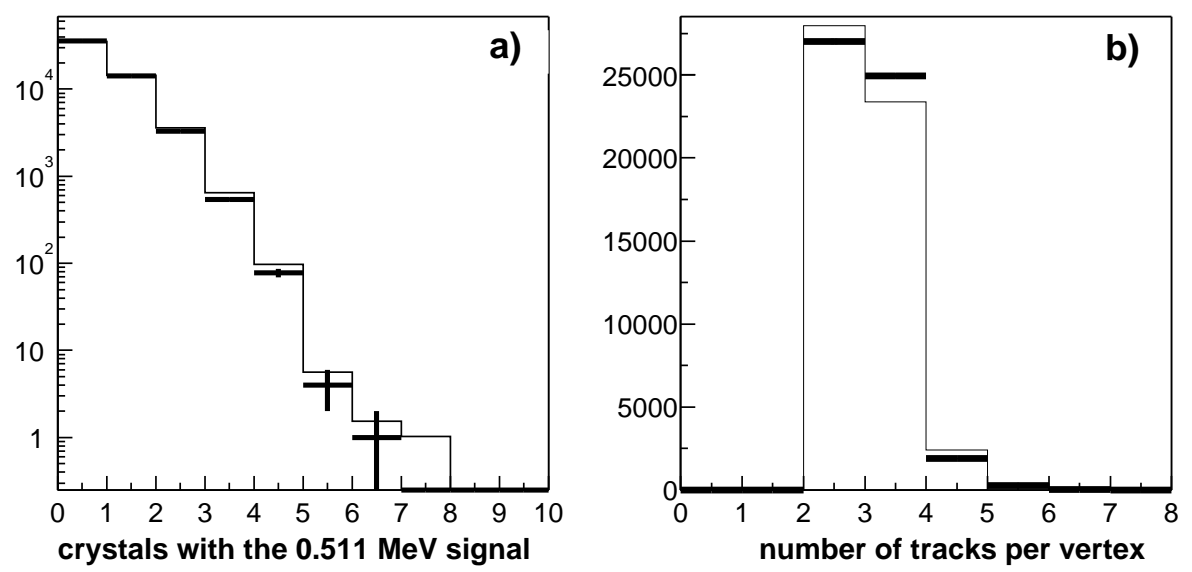

Fig. 37. Real $\bar{p}$ (bars) and MC (solid line) detector responses. a) isolated crystals with the energy response in the $511 \mathrm{keV}$ window (note the logarithmic scale). The events with 2 isolated crystals contains the entries of the opening angle histogram (see fig. 38 below); b) number of reconstructed tracks per event.
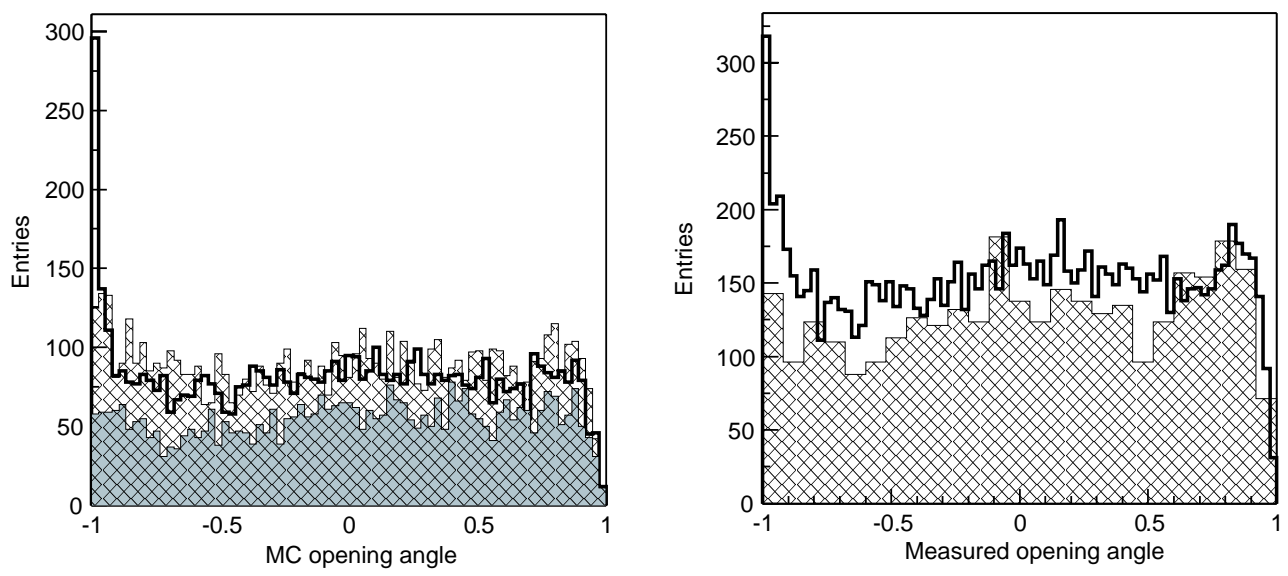

Fig. 38. Left: Monte Carlo generated $\cos \theta$ distribution of the opening angle $\theta$ for the $\bar{H}$ annihilation (bold line) and for the $\bar{p}$ annihilation background of type (1) (gray) and the uncorrelated $\bar{p}\left(e^{+} e^{-}\right)$annihilation background of type (2) (hatched) (see the text). Right: the same distribution in the case of real data during the cold mixing of $\bar{p}$ and positrons (bold line) and the same histogram (hatched) in the case of hot mixing without recombination. The $\bar{H}$ and background distributions are normalized to the same number of reconstructed vertices.

(left), where a clear signal around the value $\cos \theta \simeq-1$ appears in the case of $\bar{H}$ annihilations, in agreement with the efficiencies reported in table 3. Apart from this signal, the rest of the histogram shows a plateau of events due to the $511 \mathrm{keV} \gamma$ 's from positron annihilations that have been lost by scattering their original direction or to the accidental firing of crystals due to soft $\gamma$ 's from the e.m. cascades into the magnet or other parts of the apparatus.

During the runs, we mixed $\bar{p}$ and positrons in the recombination trap at low temperature (cold mixing) obtaining a clear $\bar{H}$ formation signal, shown with the bold 
line in fig. 38 (right). In the same figure, the hatched histogram shows the distributions obtained with the recombination completely stopped by heating the positron plasma with a radio-frequency signal [1]. The MC and real data of fig. 38 show clearly that our apparatus allows the detailed study of both $\bar{H}$ production and the various sources of background.

\section{Acknowledgements}

We would like to thank S. Bricola, P. Chiggiato, A. Mongelluzzo, G. Chuste, J. Hansen, A. Lanza, C. Marciano, J. M. Rieubland, G. Sobrero, N. Vauthier, J. CasasCubillos, M. A. Rodriguez Ruiz, B. Schmid and E. Søndergaard. Thanks are also due to the CERN AD team. Professor C. Surko provided essential advice. This work was supported by the following funding agencies: Istituto Nazionale di Fisica Nucleare (Italy), Conselho Nacional de Pesquisa e Desenvolvimento (CNPq), Fundao de Amparo a Pesquisa do Estado do Rio de Janeiro (FAPERJ) e Fundao CCMN/UFRJ (Brazil), Grant-in-Aid for Creative Basic Research of Monbukagakusho (Japan), the Swiss National Science Foundation, the Danish Natural Science Research Council, the UK Engineering and Physical Sciences Research Council, the EU (EUROTRAPS Network) and the Royal Society.

\section{References}

[1] M. Amoretti et al., Nature 419 (2002) 456.

[2] M. Niering et al., Phys. Rev. Lett 84 (2000) 5496.

[3] R. Carosi et al., Phys. Lett. B 237 (1990) 303.

[4] G. Baur et al., Phys. Lett. B 368 (1996) 251.

[5] G. Blanford et al., Phys. Rev. Lett. 80 (1998) 3037.

[6] L. S. Brown and G. Gabrielse, Rev. Mod. Phys. 58 (1986) 233.

[7] M. M. Nieto and M. H. Holzscheiter, Appl. Phys. B 60 (1995) 103.

[8] S. Maury, Hyp. Int. 109 (1997) 43.

[9] P. Riedler et al., Nucl. Instr. and Meth. A 478 (2002) 316.

[10] C. Amsler et al., Nucl. Instr. Methods A 321 (1992) 69.

[11] R. Medenwaldt et al., Nucl. Instr. and Meth. B 58 (1991) 1.

[12] M. C. Fujiwara and M. Marchesotti, Nucl. Instr. and Meth. A 484 (2002) 162. 
[13] L.K. van Geest et al., Nucl. Instr. and Meth. A 310 (1991) 261.

[14] R. DeSalvo et al., Nucl. Instr. and Meth. A 315 (1992) 375.

[15] H. Arnaudon et al.,Nucl. Instr. and Meth. A 342 (1994) 558.

[16] Delft Electronische Producten, Dwazziewegen 2, NL-9300 AB Roden, The Netherlands.

[17] ASACUSA Collaboration, private communication; M.C. Fujiwara, ATHENA Technical Report (2000) unpublished.

[18] P. Lubinski et al., Phys. Rev. Lett. 73 (1994) 3199.

[19] C. Gonzalez and F. Pedersen, Proc. of the 1999 Part. Acc. Conf. (eds. A. Luccio, W. MacKay)(IEEE, USA, 1999) p. 474.

[20] M.E. Angoletta et al., CERN report PS 2001-044 (2001).

[21] M.C. Fujiwara et al., Hyp. Int. 138 (2001) 153.

[22] H. Raimbault-Hartmann et al., Nucl. Instr. and Meth. B 126 (1997) 378.

[23] X. Huang, F. Anderegg, E. M. Hollmann, C. F. Driscoll, and T. M. O’Neil, Phys. Rev. Lett. 78 (1997) 875.

[24] C. A. Kapetanakos and A. W. Trivelpiece, J. Appl. Phys. 42 (1971) 4841.

[25] G. Gabrielse, S. Rolston, L. Haarsma and W. Kells, Phys. Lett. A 129 (1988) 38.

[26] S. L. Rolston and G. Gabrielse, Hyperfine Int. 44 (1988) 233; M. H. Holzscheiter et al., Hyperfine Int. 109 (1997) 145.

[27] L. Spitzer, in Physics of Fully Ionised Gas, (Interscience Publishers, New York, 1962) p.585.

[28] J. M. Kriesel and C. F. Driscoll, Phys. Rev. Lett. 85 (2000) 2510; R. Chu et al., Phys. Fluids B 5 (1993) 2378; C. F. Driscoll and J. H. Malmberg, Phys. Rev. Lett. 50 (1983) 167; C. F. Driscoll, K. S. Fine, and J. H. Malmberg, Phys. Fluids 29 (1986) 2015.

[29] G. Testera et al., Nucl. Instr. and Meth. A 461 (2001) 253.

[30] M. Amoretti et al., in Non Neutral Plasma Physics IV, edited by F. Anderegg, L. Schweikhard, and C. F. Driscoll, AIP Conf. Proc. 606 (2002) 45.

[31] J. D. Larson, J. C. Bergquist, W. M. Itano, and D. J. Wineland, Phys. Rev. Lett. 57 (1986) 70; T. M. O.Neil, Phys. Fluids 24 (1981) 1447.

[32] L. Turner, Phys. Fluids. 30 (1987) 3196.

[33] D. H. E. Dubin, Phys. Rev. Lett. 66 (1991) 2076.

[34] M. D. Tinkle, R. G. Greaves, C. M. Surko, R. L. Spencer, and G. W. Mason, Phys. Rev. Lett. 72 (1994) 352.

[35] D. H. E. Dubin, Phys. Fluids B 5 (1993) 295. 
[36] M. Amoretti et al., Phys. Rev. Lett. 91 (2003) 055001.

[37] M. Amoretti et al., Phys. Plasmas 10 (2003) 3056.

[38] C. M. Surko, S. J. Gilbert, and R. G. Greaves, in Non-Neutral Plasma Physics III, edited by J. J. Bollinger, R. L. Spencer and R. C. Davidson, AIP Conf. Pro. 498 (1999) 3.

[39] T. J. Murphy and C. M. Surko, Phys. Rev. A 46 (1992) 5696.

[40] R. G. Greaves, M. D. Tinkle and C. M. Surko, Phys. Plasmas 1 (1994) 1439.

[41] C.F. Driscoll and J.H. Malmberg, Phys. Rev. Lett 50 (1983) 167.

[42] E.M. Hollmann, F. Anderegg, and C.F. Driscoll, Phys. Plasma 7 (2000) 2776.

[43] A.P. Mills Jr. and E.M. Gullikson, Appl. Phys. Lett. 49 (1986) 1121.

[44] R. Khatri, M. Charlton, P. Sferlazzo, K.G. Lynn, A.P. Mills Jr., and L.O. Roellig, Appl. Phys. Lett. 57 (1990) 2374.

[45] J.P. Merrison, M. Charlton, B.I. Deutch, and L. V. Jørgensen, J. Phys: Condens. Matter 4 (1992) L207.

[46] L. V. Jørgensen et al., in Nonneutral Plasma Physics IV, (F. Anderegg, L. Schweikhard, and C. F. Driscoll, eds.), AIP Conf. Proc. 606 (2002) 35.

[47] L. V. Jørgensen, M. J. T. Collier, K. S. Fine, T. L. Watson, D. P. van der Werf, and M. Charlton, in Positron Annihilation ICPA-12, edited by W. Triftshäuser, G. Kögel and P. Sperr, Mat. Sci. Forum 363-365 (2001) 634.

[48] M. J. T. Collier, L. V. Jørgensen, O. I. Meshkov, D. P. van der Werf, and M. Charlton, in Non-Neutral Plasma Physics III, edited by J. J. Bollinger, R. L. Spencer and R. C. Davidson, AIP Conf. Proc. 498 (1999) 13.

[49] R. G. Greaves and C. M. Surko, Phys. Rev. Lett. 85 (2000) 1883.

[50] R. G. Greaves and C. M. Surko, Phys. Plasmas 8 (2001) 1879.

[51] G.R. Heyland, M. Charlton, T.C. Griffith, and G.L. Wright, Can. J. Phys. 60 (1982) 503.

[52] M. Charlton and J.W. Humberston, Positron Physics, Cambridge University Press, (2000) chapter 6.

[53] C. Regenfus, Hyp. Int. 119 (1999) 301.

[54] C. Regenfus, Nucl. Instr. and Meth. A 501 (2003) 65.

[55] C. Amsler et al., Nucl. Instr. and Meth. A 480 (2002) 494.

[56] O. Toker et al., Nucl. Instr. and Meth. A 340 (1994) 572.

[57] C. Amsler et al., Nucl. Instr. and Meth. A 480 (2002) 501. 
[58] Rene Brun and Fons Rademakers, ROOT - An Object Oriented Data Analysis Framework, Proc. AIHENP'96 Workshop, Lausanne, Sep. 1996, Nucl. Instr. and Meth. in Phys. Res. A 389 (1997) 81. See also http://root.cern.ch/.

[59] CERN Application Software Group, GEANT: Detector Description and Simulation Tool, CERN Program Library W5013, Geneva, 1993. See also http://wwwinfo.cern.ch/asd/geant/

[60] R. Armenteros and B. French in $\bar{N} N$ interactions in high energy physics 4 (1969) 237, E.H.S. Burhop ed., Academic Press.

[61] G. Bendiscioli and D. Kharzeev, Riv. Nuovo Cim. 17, n.6 (1994) 1.

[62] E. Calligarich et al., Nucl Instr. and Meth A311 (1992) 151.

[63] M. Amoretti et al., submitted to Phys. Rev. Lett. 